Ann. Zootech., 1978, 27 (3), 377-408.

\title{
Organisation de la motricité de l'intestin grêle chez le porc et influence de l'alimentation
}

\author{
J. P. LAPLACE \\ avec la collaboration technique de C. Grirman \\ Laboratoire de Physiologie de la Nutrition, \\ Centre national de Recherches zootechniques, I.N.R.A., \\ 78350 Jouly-en-Josas (France)
}

\begin{abstract}
Résumé
Une étude systématique des caractéristiques des complexes myoélectriques de l'intestiu grêle en fonction de l'alimentation a été réalisée chez ro porcs de race Large White. Les électroinyogrammes intestinaux ont été recueillis à partir d'électrodes chroniques souples implantées préalablement sous anesthésie générale. Les enregistrements ont été effectués dans tous les cas après habituation aux $z$ types de régimes utilisés chez chacun des animaux : aliment standard de croissance et aliment semi-synthétique contenant $8 z$ p. I oo d'amidon de maïs purifié. Le rythme (le distribution en 2 repas à $9 \mathrm{~h}$ et $\mathrm{I} 6 \mathrm{~h}$ 3o a été uniformément appliqué.

Deux à trois complexes myoélectriques sont en permanence présents au long de l'intestin grêle du Porc. Pour un régime standard, on en dénombre en moyenne, par 24 h, 20,4 au niveau duodénal, I6,6 sur le jéjunum et I I, I sur l'iléon, 40 p. 100 d'entre eux ne franchissant pas la totalité du viscère. Ces nombres, stables pour un site donné chez un même animal, sont très variables d'un porc à l'autre. L'intervalle moyen de récurrence est de l'ordre de $70 \mathrm{mn}$ au niveau duodénal, I Io à r $20 \mathrm{mn}$ au niveau iléal. En dépit de diverses irrégularités de la migration des complexes, celle-ci s'effectue à des vitesses moyennes de l'ordre de $30 \mathrm{~cm} / \mathrm{mn}$ sur le duodénum, Io $\mathrm{cm} / \mathrm{mnn}$ sur le jéjunum et $5 \mathrm{~cm} / \mathrm{mn}$ sur l'iléon. La prise de nourriture est responsable de l'apparition d'une organisation postprandiale particulière durant environ $2 \mathrm{~h}$. Au cours de cette période, la phase de quiescence est abolie; l'activité irrégulière intense est prédominante sans effacer cependant les activités régulières. L'augmentation du degré de réplétion intestinale se traduit notamment par l'intensification des activités irrégulières; à l'inverse, la durée de la période de quiescence augmente au cours du repos alimentaire nocturne.

Par rapport à ces données, la consommation de l'aliment semi-synthétique conduit à enregistrer en moyenne par $24 \mathrm{~h}, \mathrm{i} 8,5$ complexes myoélectriques au niveau duodénal et i 3,7 au niveau iléal. Il en résulte une récurrence iléale à intervalles plus brefs (90 à i I o mn). Mais 50 p. roo des activités régulières iléales ne sont pas l'aboutissement d'une migration proximo-distale continue. Dans ce sens, la migration des complexes myoélectriques apparaît relativement désorganisée lors d'ingestion de l'aliment amidon de maîs par rapport à ce qui est observé avec le régime standard. Parallèlement, sont mesurées des vitesses de migration sensiblement plus faibles $(\mathrm{I} 6,4 \mathrm{~cm}$. mn au niveau du quart proximal de l'intestin grêle). Ces particularités pourraient être la conséquence de la composition de l'aliment, hypothèse discutée en relation avec l'absorption des produits de la digestion de l'amidon de maïs. Enfin, l'allongement impor tant de la phase de quiescence entre le duodénum et l'iléon, au détriment de la phase d'activité irrégulière, peut être relié à la moindre réplétion digestive (à ingéré égal) du fait de la très haute digestibilité du régime anidon de maïs.
\end{abstract}




\section{Introduction}

L'enregistrement simultané de l'électromyogramme en divers sites échelonnés au long de l'intestin grêle du chien à jeun a conduit Szurszewssi (I969) à décrire l'apparition périodique sur le duodénum de phases d'intense activité, chacune d'entre elles faisant l'objet d'une lente migration en direction aborale. Cette figure constitue l'une des phases d'une séquence de phénomènes, soumise à récurrence cyclique, connue sous le nom de migrating myoelectric complex (MI.M.C.) (CODE et MARI,E'TT, I975).

Un complexe myoélectrique comporte successivement trois phases. Chacune répond à un type de structuration des activités électriques auquel correspond ur aspect particulier des mécanogrammes. Ce sont respectivement :

- Phase I : - absence de potentiels d'action surimposés aux ondes lentes;

- absence de variations de la pression endoluminale;

- usuellement dénommée phase de quiescence.

- Phase II : - présence de bouffées de potentiels d'action d'intensité variable surchargeant irrégulièrement un certain nombre d'ondes lentes;

- fluctuations rapides, d'amplitude variable, de la pression endoluminale (Bueno, Fioramontr et Ruckebusch, 1975); ces pics de pression répondent aux bouffées de potentiels d'action;

- usuellement dénommée activité de pointes irrégulière.

- Phase III : - présence systématique durant plusieurs minutes, sur chacune des ondes lentes consécutives, de bouffées très fournies de potentiels d'action d'amplitude maximale; cette organisation des activités électriques correspond à la figure décrite par SZURSZEWSKI (I969);

- élévation soutenue de la pression endoluminale moyenne supportant des pics de pression (BUENo, FIORAMONTI et RUCKEBUSCH, I975);

- usuellement dénommée activité de pointes régulière.

Une phase IV est identifiée par CODE et MARLETT (I975) comme la décroissance de la phase III et le prélude au silence moteur. Les dénominations communément adoptées sont fondées sur la chronologie d'apparition des bouffées de potentiels d'action (générés pour l'essentiel par la couche musculaire circulaire) par rapport au rythme à ondes lentes (issues de la couche longitudinale).

L'enchaînement séquentiel de ces 3 phases est caractéristique de 1'organisation de la motricité de 1'intestin grêle de plusieurs espèces animales. Parmi celles-ci, il existe cependant des différences dans l'in fluence exercée par la prise de nourriture sur cette organisation en complexes myoélectriques.

Amplement confirmée chez le Chien, elle n'existe en effet dans cette espèce que chez le sujet à jeun. La première observation, chez le Mouton, de cette succession de phénomènes mécaniques et électriques en un même site intestinal (RUCKËBUSCH et LAPIACE, I967; LAPLACE, I968 $\left({ }^{*}\right)$ ) a été confirmée par la description de GRIVEI, et RUCKeBusch (I972) démontrant l'existence des complexes, chez le Mouton ainsi que chez le Lapin, même chez l'animal alimenté ad libitum. Il en est de même

(*) In LaPLACE (1968):

Pp. 63-64: activités mécaniques.

Pp. $84-85$ : activités électriques. 
pour le Veau (RUCKebusch, Dardiliat et Hatey, I972). Par contre, et à l'instar du Chien, la prise de nourriture supprime les complexes myoélectriques chez le Rat (RUCKebusch et Ferre, I973) et l'Homme (Heldemans et al., I977).

Dans le cas du Porc, RuckEBusch et BuExo (I976) ont étudié plus particulièrement, chez des porcs miniatures adultes, l'in fluence de la prise de nourriture sur les activités électriques gastro-intestinales, respectivement dans des situations d'alimentation ad libitum et de distribution de I ou 2 repas par jour. Une description des complexes myoélectriques au niveau du seul intestin grêle proximal a par ailleurs été fournie par LAPLACE et GERMAIN (I976) chez des porcs en croissance de race Large White. Étant donné l'importance des problèmes nutritionnels et de la pathologie digestive chez ces derniers, l'objet du présent travail est d'apporter une description aussi complète que possible des complexes myoélectriques tout au long de l'intestin grêle, et de leurs variations normales pour des régimes standard ou semi-synthétique.

\section{Matériel et méthodes}

\section{Animaux et préparation chirurgicale}

Dix pores de race Large White d'un poids vif initial de $59,7 \pm 2,6 \mathrm{~kg}$, codés A, B, C, I) E, F, G, H, I et M, ont été utilisés pour cette étude. Leur gain moyen quotidien individuel de poids vif au cours de leur vie expérimentale a été compris entre 353 et $795 \mathrm{~g}$ par jour (moyenne générale : $55^{2} \pm 46 \mathrm{~g}$ par jour). Ils ont été équipés d'électrodes chroniques à la faveur d'une anesthésie générale, induite par les barbituriques en injection endoveineuse rapide et entretenue par le méthoxyflurane appliqué au masque (vecteurs $\mathrm{O}_{2}-\mathrm{N}_{2} \mathrm{O}$ ). Les électrodes sont constituées de brins d'acier inoxydable (diamètre $\mathbf{I} 20 \mu \mathrm{m}$, longueur $220 \mathrm{~cm}$ ), électriquement isolés et associés par trois au sein d'une gaine de silicone médical (Silastic ${ }^{\mathrm{R}}$ ou Rhodorsi1 ${ }^{\mathrm{R}}$ ). A la faveur d'une laparotomie dans le creux du flanc droit, elles ont été implantées dans la paroi de l'intestin grêle et éventuellement de l'antre gastrique à raison de 3 en chaque site (distances inter-électrodes $2 \mathrm{~mm}$ ).

Le nrode d'implantation est celui utilisé depuis r 966 chez le Mouton (RUCKEBUSCH et LAPLACE, I 966; Laplace, 1968) et précisé dans le cas particulier du Porc (LAPLACE, I972). La préparation ainsi obtenue permet d'enregistrer 1'électromyogramme global (E.M.G.) de la musculature lisse intestinale selon les modalités explicitées par RUCKEBUSCH (1972, I973).

Après réalisation des expériences prévues, les porcs ont été abattus. L'intestin grèle a fait aussitôt l'objet de mensurations : longueur totale et localisation exacte des sites d'électrodes. Pour permettre la systématisation des résultats et faciliter les comparaisons entre porcs en dépit des variations entre individus (longueur totale de l'intestin छrêle variant entre $\mathrm{I} 3$ et I $8 \mathrm{~m}$ chez le porc de $60 \mathrm{~kg}$ ), ces mesures post mortem ont été converties en pourcentages. La localisation de chaque site d'électrodes est alors exprimée par le pourcentage de la longueur totale de l'intestin grêle que représente la distance qui sépare ce site du pylore. Le tableau I récapitule sur cette base les diverses préparations utilisées.

Les vitesses de propagation ont été déterminées dans tous les cas sur les distances entre sites relativement rapprochés $(5$ à $15 \mathrm{~cm})$. A partir de ces mesures, l'estimation par le calcul d'une vitesse moyenne de propagation au long d'un territoire plus vaste (quart proximal de l'intestin grêle ou łotalité de celui-ci) a eu re. cours au calcul de la moyenne harmonique des valeurs mesurées en plusieurs sites de ce territoire. 


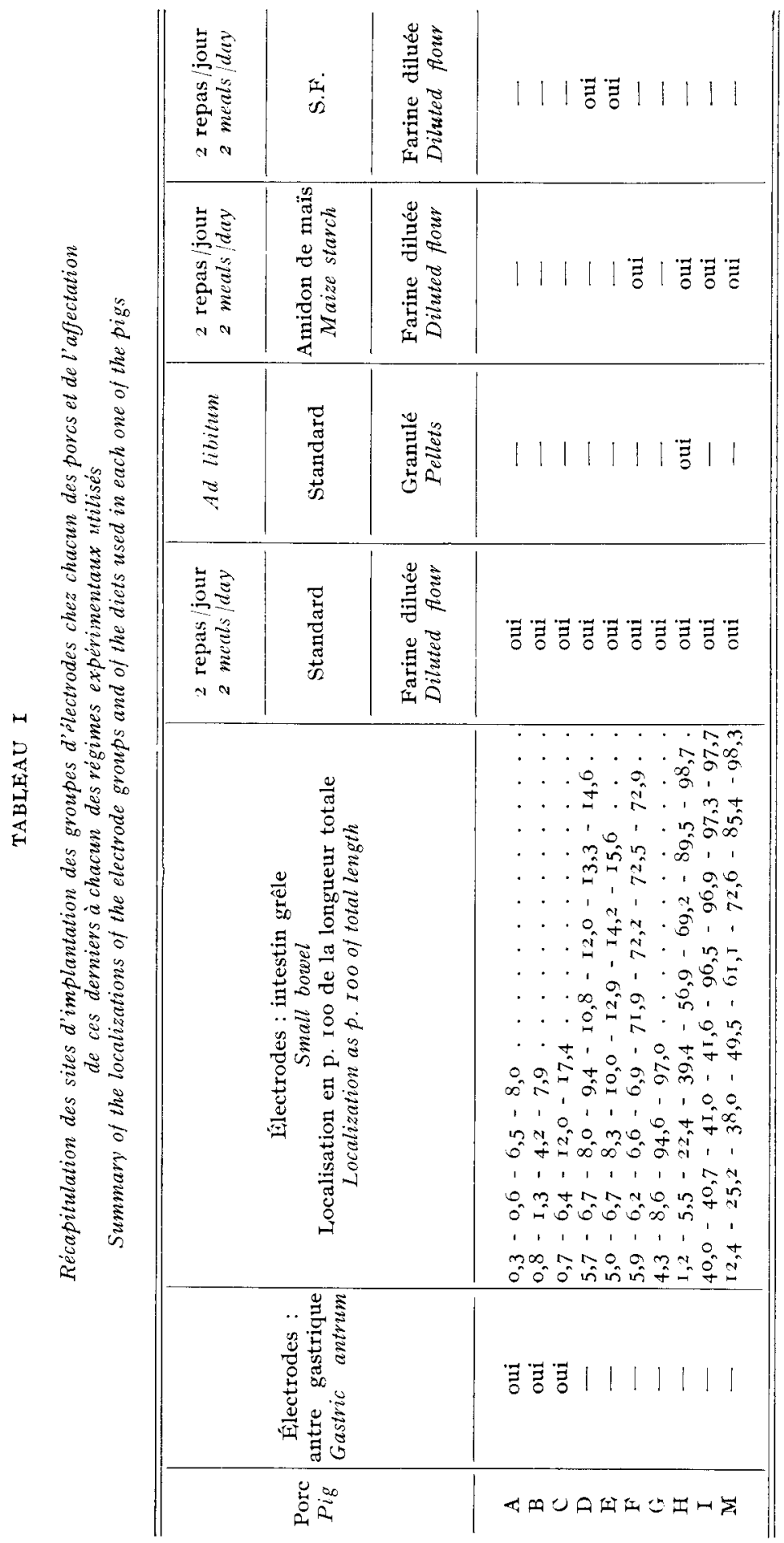




\section{Aliments et modes de distribution}

L'alıment de référence, dont la composition centésimale est indiquée dans le tableau 2, est un aliment standard de croissance ( $88 \mathrm{p}$. Ioo de matière sèche) auquel les Porcs sont accoutumés depuis le sevrage. Il a été distribué systématiquement à raison de 2 repas par jour ( 9 h et $I 6$ h 30 ) sous forme de soupe après dilution far 2 fois son poids d'eau. Ainsi I repas de I $\mathrm{kg}$ de farine fraîche représente 1 'ingestion de $3 \mathrm{~kg}$ de soupe. Chez un porc (H) le même aliment présenté sous forme de granulé a été laissé à disposition permanente de l'animal en quantité excessive. L'accoutumance à cette situation d'ad libitum, préalable à tout enregistrement, a été de io jours.

Deux aliments semi-synthétiques dont la composition est indiquée dans le tableau 2 ort par ailleurs été utilisés. Ils sont respectivement dénommés « Amidon de mais " et "S.F. ". Ce dernier aliment a pour originalités essentielles sa granulométrie très fine (particules de $50 \mu \mathrm{m}$ ) et l'usage de caséine comme source d'azote. Ces 2 régimes ont été distribués dans tous les cas 2 fois par jour sous forme de soupe après dilution par I fois leur poids d'eau seulement, en raison de la fluidité

\section{TABLEAU 2}

Composition centésimale des aliments utilisés

Percentual composition of the feeds used

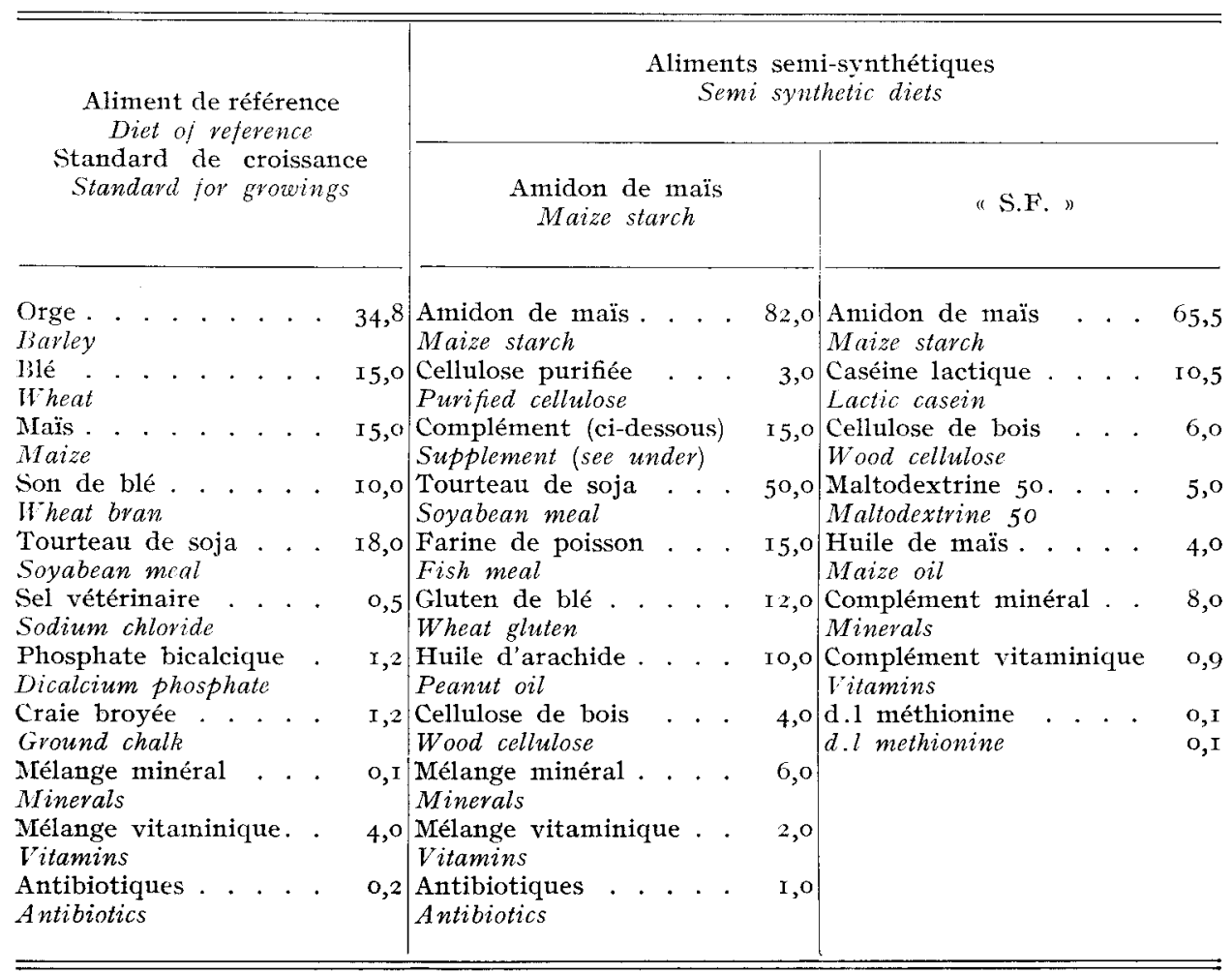


de la soupe obtenue. I,es en registrements avec l'un ou l'autre de ces régimes n'ont été réalisés qu'après des périodes d'accoutumance de ro jours.

Les porcs ont disposé en permanence d'eau à volonté. Leurs affectations. respectives aux différents régimes sont récapitulées dans le tableau $I$.

\section{Conditions expérimentales}

L,es porcs, logés au sol individuellement jusqu'à l'opération, ont été installés, aussitôt après celle-ci, dans une cage du type cage à métabolisme flacée dans une salle isolant l'animal des bruits extérieurs, comportant une cage de Faraday complè te dans l'épaisseur des parois et dont la température, la ventilation et l'éclairage(jour-nuit à $7 \mathrm{~h}$ et Ig h) sont contrôlés automatiquement. Une surveillance par circuit de télévision a évité toute présence humaine en dehors de la distribution des repas et de l'enlèvement des déjections assurés simultanément. Les électrođes, supportées par un système de balancier accompagnant les mouvemients de l'animal, or,t été connectées directement à la plaque d'entrée de l'enregistreur situé luimême en dehors de la salle d'isolement.

\section{Enregistrements}

Après réhabilitation post opératoire et accoutumance au nouvel environnement durant 8 jours, les activités électriques intestinales ont été recueillies pendant des périodes continues de 24 ou $4^{8}$ heures sur un polygraphe 8 voies (Dynograph RM Beckman), par l'intermédiaire de coupleurs d'entrée AC/DC (type 9806 A, Beckman). Ces coupleurs autorisent 1'utilisation de constantes de temps très brèves $(0,03 \mathrm{~s})$, ce qui permet de réduire au minimum les fluctuations du tracé liées aux ondes lentes du rythme électrique de base. Les variations électriques rapides (potentiels d'action) ont été enregistrées selon 3 modalités :

- Inscription directe sur papier à des vitesses de défilement de 2,5 ou $5 \mathrm{~cm} /$ $m n$ des activités recueillies en 8 sites au plus simultanément.

$O u$ : sommation des activités dérivées en 4 sites par unité de temps de io à $90 \mathrm{~s}$ (le plus souvent $60 \mathrm{~s}$ ) à l'aide d'un intégrateur linéaire (LATOUR, I973) à 4 voies. L'inscription est alors effectuée sur 4 voies du polygraphe. Les 4 autres sont réservées au tracé E.M.G. direct parallèle des 4 mêmes sites, ce qui autorise le contrôle permanent des phénomènes.

Et/ou : sommation comme ci dessus, les signaux de sortie de l'intégrateur faisant l'objet d'une conversion en mode digital (FAYART, DABURON et LAPIACE, I978) au terme de chaque unité de temps. Pour chacun des 4 sites étudiés, la valeur numérique exprimant, dans une échelle arbitraire o à 99 , la somme des activités rapides intestinales dans l'intervalle de temps considéré est transcrite $24 \mathrm{~h}$ sur 24 sur une imprimante (IBM Selectric type 73). Ce dernier procédé, simple extension de l'analyse quantitative proposée par LATOUR (I973), autorise le calcul de l'intensité moyenne des périodes d'activité de pointes régulière ou irrégulière. Ce processus a été appliqué pour faciliter l'expression graphique de l'organisation de la motricité intestinale durant un cycle de $24 \mathrm{~h}$. Il permet éventuellement la comparaison des intensités d'activité au long d'un nycthémère, ou entre 2 nycthémères consécutifs pour des régimes différents. Mais cette approche commode ne saurait être appliquée à des intervalles de temps beaucoup plus grands, en raison de l'évolution des élec- 
trodes (RUckeвusch, I973) : réaction fibro-séreuse précoce puis développement lent du processus de rejet qui conduit à les chasser hors de la musculature intestinale.

\section{Résultats}

I. - Caractéristiques des complexes myoélectriques chez le Porc recevant 2 repas par jour d'aliment standard

\section{a) Description générale}

La description des complexes myoélectriques, telle qu'elle a été précédemment rappelée dans le cas du Chien, est entièrement applicable au Porc (fig. I). La phase I de quiescence est caractérisée par l'absence quasi-totale de potentiels d'action surchargeant les ondes lentes. La phase II d'activité de pointes irrégulière correspond à la présence de bouffées de pointes surchargeant les ondes lentes de façon irrégulière et apparemment aléatoire. Cependant, la fréquence d'apparition des bouffées et l'amplitude des potentiels de pointe qui les constituent, augmentent progressivement au fil du déroulement de la phase II. La phase III, d'activité de pointes régulière, est définie par la présence systématique de bouffées de potentiels très fournies surchargeant chaque onde lente. Durant cette phase III, l'activité E.M.G. de la musculature intestinale est maximale comme le traduit clairement l'intégration des activités rapides.

Cette séquence est observable dès le duodénum proximal, où l'apparition de la phase III et la période consécutive de quiescence coïncident systématiquement avec une suspension de l'activité contractile antrale (fig. 2). La succession de ces 3 phases se répète indéfiniment à intervalles réguliers au niveau du duodénum proximal, chaque séquence consécutive balayant l'intestin grêle en une lente migration dans le sens ora1-aboral (fig. 3).

\section{b) Périodicité et durée des phénomènes}

Le nombre total de séquences (I + II + III) enregistrées au cours des $24 \mathrm{~h}$ est en moyenne de 20 au niveau du quart proximal de l'intestin grêle d'un porc consommant un régime standard. Il est en moyenne, respectivement au niveau du jéjunum et au niveau du quart distal de l'intestin grêle, de l'ordre de I 6 et de II (tabl. 3). Cette téduction correspond à un pourcentage de complexes ne franchissant pas la totalité de 1'intestin grêle d'environ $40 \mathrm{p}$. Ioo (estimation globale sur la base des moyennes).

Les valeurs enregistrées en ces différents sites d'intestin grêle varient peu chez un même porc. Par contre, la variabilité entre animaux est importante. Des nombres, retrouvés individuellement de façon stable chez chaque porc, et aussi différents que 26 à 27,23, I 8 à I 9 , ou I 5 à I 6 , sont enregistrés au niveau duodénal chez nos différents animaux.

Cette stabilité individuelle du nombre moyen de complexes enregistrés en un site donné implique une récurrence cyclique des phénomènes. La périodicité des complexes, appréciée par le début parfaitement bien marqué des phases d'activité régulière, est observée systématiquement. Les intervalles (durée des complexes ou période des phénomènes) sont de $70 \mathrm{mn}$ environ pour le quart proximal de 1'intestin grêle, et de $80 \mathrm{mn}$ au niveau des $50 \mathrm{p}$. Ioo médiatıs de l'intestin grêle 


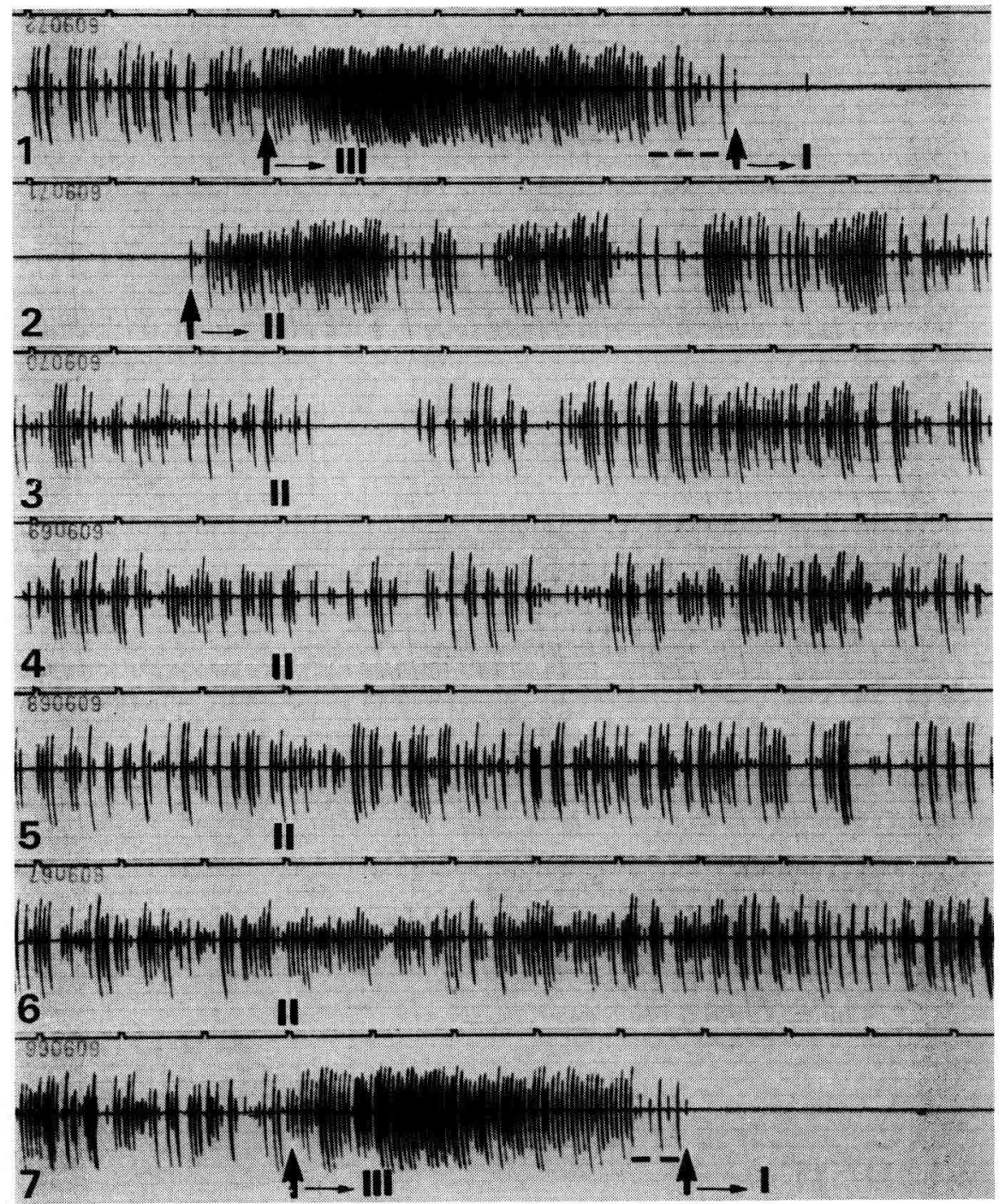

Ií. I. - Électromyogramme duodénal global, dérivé à I6 $\mathrm{cm}$ du pylore chez un porc consommant un régime standard. I.es 7 bandes sont vigoureusement consécutives dans le temps, et illustrent le déroulement complet d'un complexe myoélectrique en un même site intestinat.

En I, une phase d'activité de pointes réguliève (phase III) duve $4 m n$ zo. Elle est suivie, après une période intermédiaire (pointillés) équivalant à la phase IV décrite par CODE et MARLETT (I975) chez le Chion, par une période de quiescence (phase I) durant un peu plus de 5 mn. Puis on observe le dévoulement, jusqu'au début du tracé 7 , de la phase II d'activité de pointes irvégulière. Enfin est envegistrée une nouvelle phase III (4 mn) suivie de quiescence. I.a séquence complète du début de la phase I à la fin de la phase III dure $70 \mathrm{mn}$.

La base de temps accompagnant chaque bande indique les minutes.

Elecromyographic recording of duodenal activity $16 \mathrm{~cm}$ distal to the pylorus in a pig fed a standard diet. The 7 panels, from top to bottom, ave consecutive and show the full display of a migrating myoelectrical complex in the same intestinal site.

Panel $I$ shows a regular spiking activity (phase III) lasting $4 \mathrm{mn} 30$. It is followed, by an intermediate period (dotted line) analogous to phase IV described by CODE and MARLETT (I975) in the Dog, and then by a quiescent phase (I) lasting more than $5 \mathrm{mn}$. Thereatter an irregular 


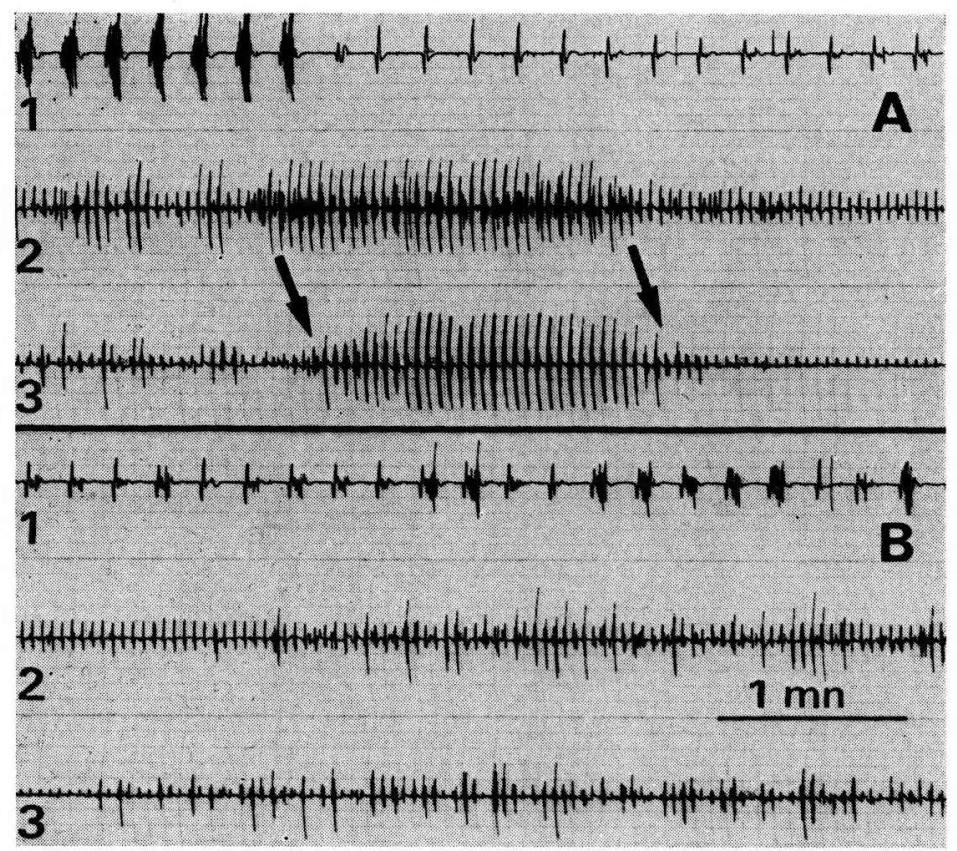

FIG. 2. - Electromyogramme de l'antre pylorique (I) à $2 \mathrm{~cm}$ du pylore, et du duodénum respectivement à 13 et $24 \mathrm{~cm}$ en aval du pylove (2 et 3) chez le Porc. Les tracés de la partie $B$ sont immédiatement consécutifs aux tracés homologues de la partie $A$ sur laquelle on note la naissance et la propagation d'une phase d'activité régulière, concomitantes de la suspension des activités rapides antrales.

Electromyographic activities of pyloric antrum $(I) 2 \mathrm{~cm}$ orad to the pylorus, and of duodenum 13 and $24 \mathrm{~cm}$ distal to the pylorus, respectively $(2$ and 3$)$ in the $P$ ig. The $B$-records are immediately consecutive to the homologous A-records where the first appearance and propagation of a duodenal regular spiking activity is accompanied by an interruption of antral spike bursts.

(fig. 4). Ils atteignent I Io à I $20 \mathrm{mn}$ au niveau distal. Cependant, des variations assez larges autour des temps moyens de récurrence peuvent résulter d'anomalies occasionnelles de la migration des complexes.

Indépendamment de cet aspect particulier, la mesure de la durée moyenne des 3 phases constitutives des complexes myoélectriques montre (tabl. 3) que les périodes d'activité de pointes régulière (III) ont une durée stable quel que soit le site intestinal. L'augmentation de la durée du cycle entre le duodénum et l'iléon, de l'ordre de $4^{0} \mathrm{mn}$ en régime standard, est liée à un allongement égal d'environ $20 \mathrm{mn}$ des phases de silence (I) et d'activité irrégulière (II).

\section{c) Migration des complexes myoélectriques : caractéristiques et anomalies}

L'enregistrement simultané des électromyogrammes en divers sites régulièrement échelonnés tout au long de l'intestin grêle montre (fig. 5) que, dans le cas

spiking phase (II) takes place until the beginning of the 7 th panel of the record. Lastly another phase (III) (4 mn) is recorded and followed by quiescence. The full sequcnce from the beginning of phase $I$ to the end of phase III lasts $70 \mathrm{mn}$.

The time marker on each record panel indicates the minutes. 


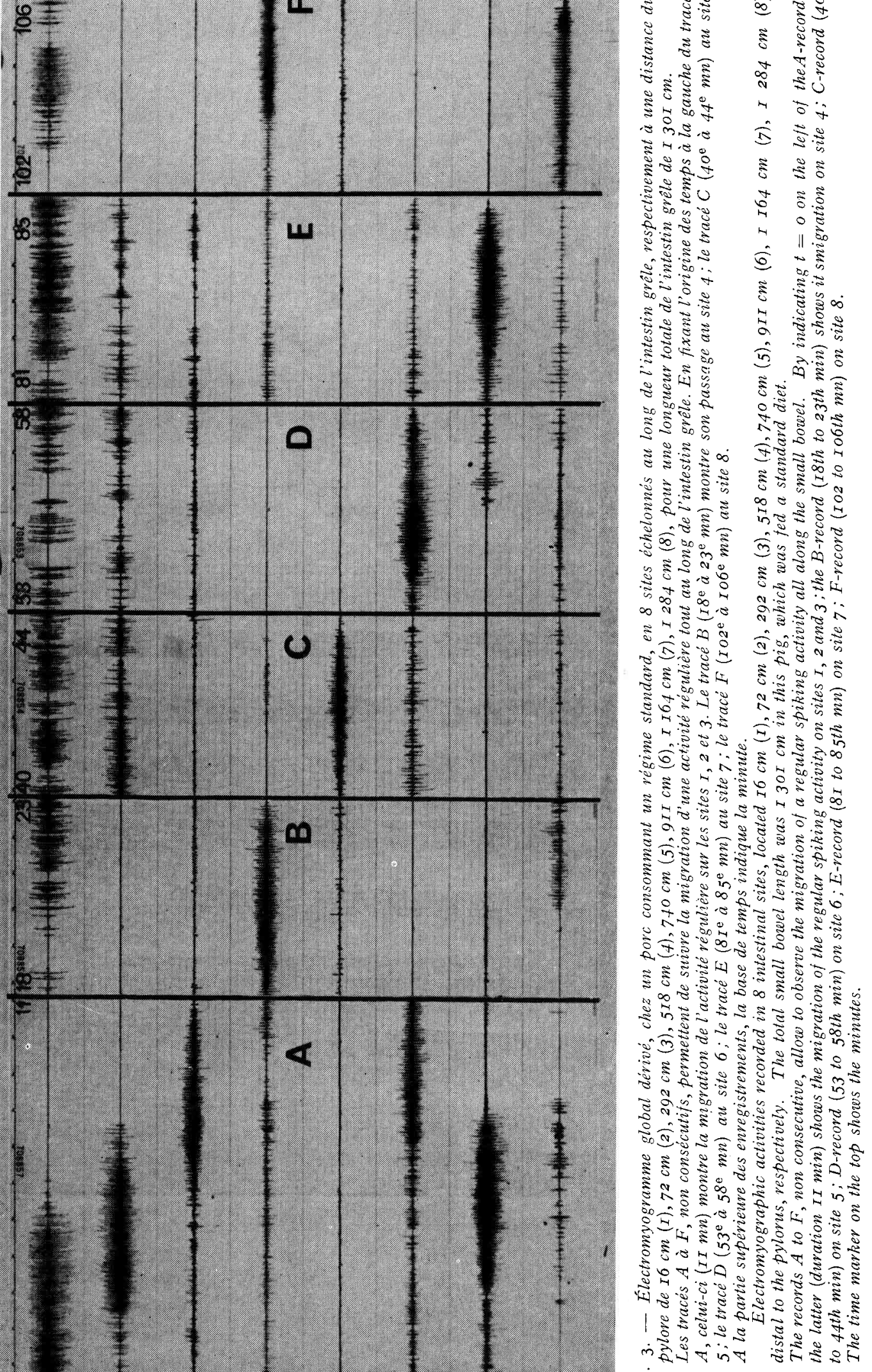


MOTRICITÉ INTESTINALE CHEZ LE PORC

$\approx \underbrace{2}_{\infty}=\overline{8}$

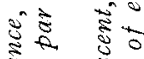

范

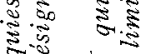

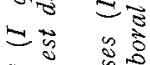

₹.

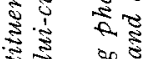

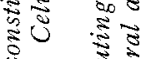

8.5

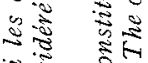

है

के

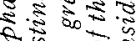

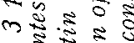

ง. हैँ

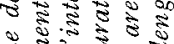

ํำ

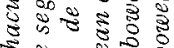

$5 \approx$ 노유

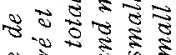

\& 3 है है के के

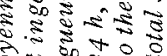

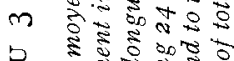

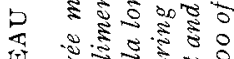

4

क

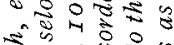

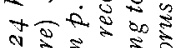

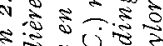

ริำ 눈 50

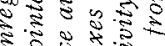

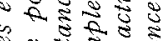

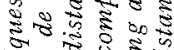

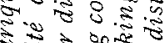

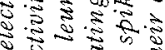

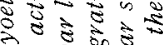

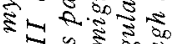

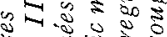

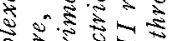
ㄴำ

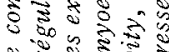
8 ํํำ ร. ำ ㅇำ ริำ हैํำ ํำ

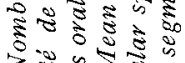
政

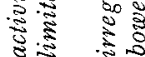

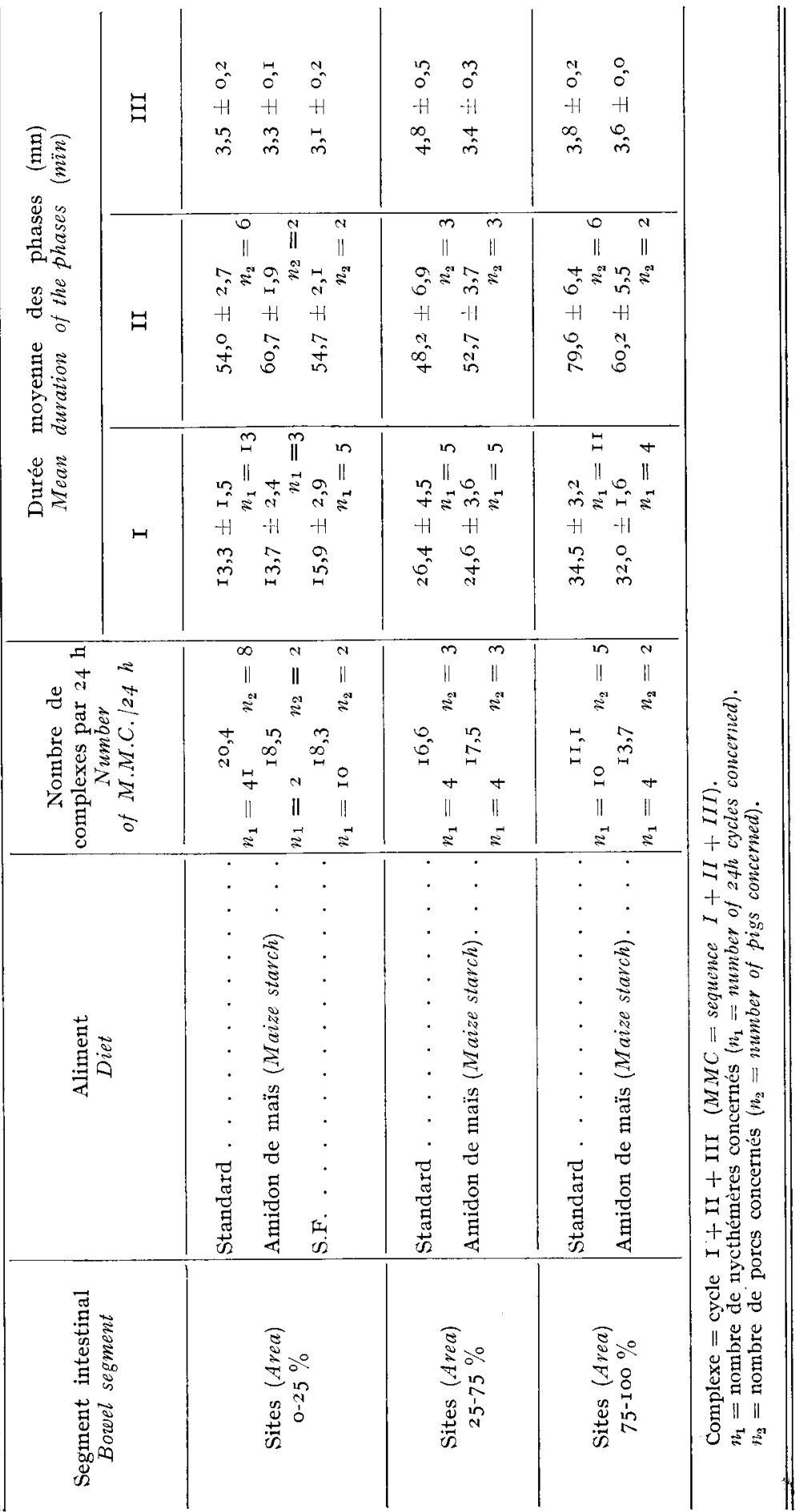




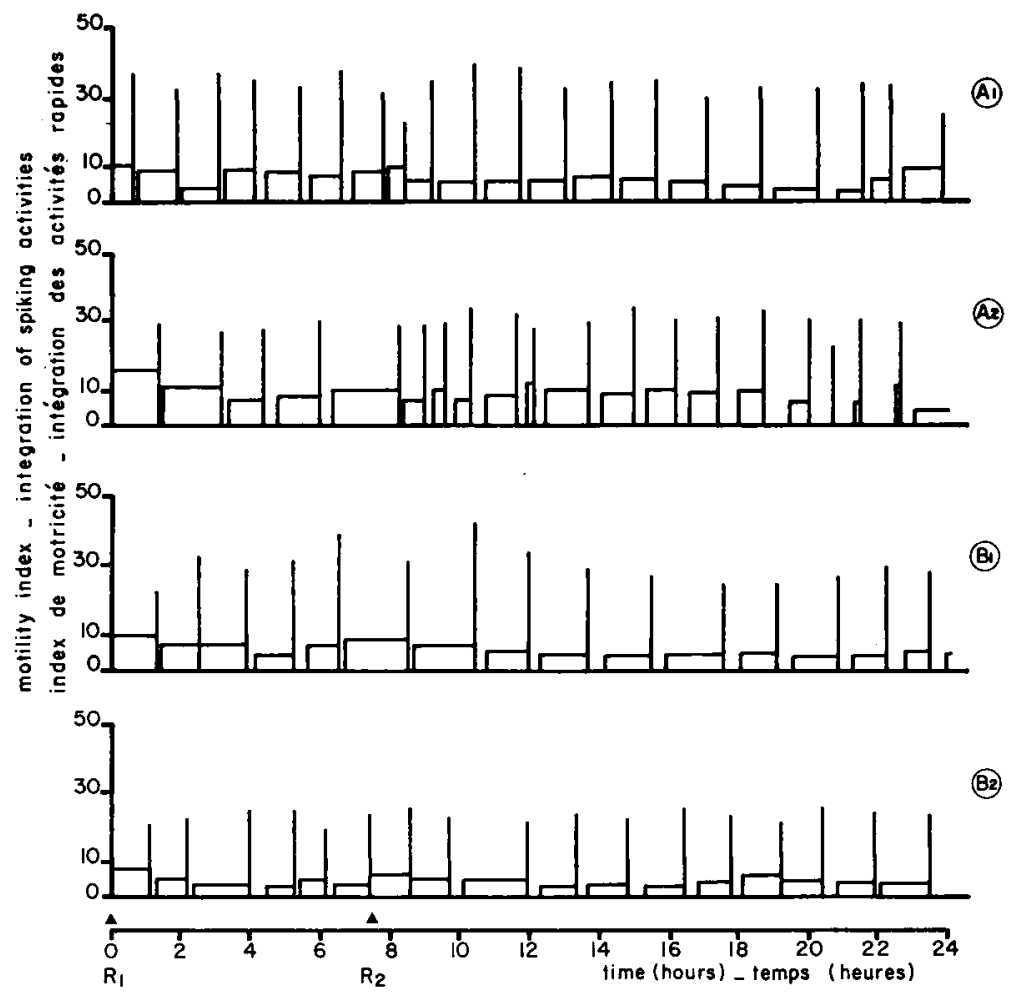

Fic. 4. -..- Représentation graphique de la succession des complexes myoélectriques au cours d'un cycle de 24 h au niveau du duodénum (à IfI cm en aval du pylove - AI et A2) et du jéjunum (à $5 \mathrm{I} 8 \mathrm{~cm}$ du pylove - BI et Ba) chez le Pớc selon la nature de l'aliment ingéré $(A I$ et $B I=$ standard, $A \mathbf{2}=$ "S.l. ", $\mathrm{B2}=$ amidon de maïs) à raison de $I 200 \mathrm{~g}$ de farine lors de chacun des 2 repas quotidiens ( $R T$ à $9 h$ oo - R2 à I 6 h 30 ). I.a hauteur des rectangles (activités irrégulières) et celle des barres (activités régulières) est proportionnelle à la hauteur moyenne des pics d'intégration exprimant l'activité intestinale pendant la durée de la phase considévée.

Pour chacun de ces graphiques, le nombre de séquences par $2+h$, et la durée moyenne des phases $I, I I, I I I$ sont respectivement dans l'ovdre :

$$
\begin{aligned}
& \text { A I : } n=20 \quad \mathrm{I}=\mathrm{I} 7, \mathrm{I}+2, \mathrm{O} \quad \mathrm{II}=54, \mathrm{I}: 2,8 \quad \text { III }=3,5 \pm 0, \mathrm{I} \mathrm{mn} \\
& \text { A2 : } n=\text { I9 } \quad \text { I }=25,5 \text { - } 3,2 \quad \text { II }=48,9 \text { - } 7,0 \quad \text { III }=3,8 \text { o, I } \mathrm{mn} \\
& \mathrm{BI}_{\mathrm{I}}: n=\mathrm{I} 5 \quad \mathrm{I}=\mathrm{I} 8,6+2,4 \quad \text { II }=73,3 \cdots 5,7 \quad \text { III }=3,9 \pm 0, \mathrm{I} \mathrm{mn}
\end{aligned}
$$

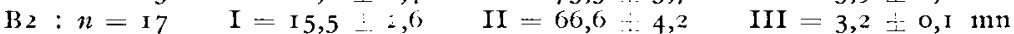

(iraph of the migrating myoelectrical complexes during a $2 f^{h}$ cycle on the duodenum (I $1 \mathrm{I} \mathrm{cm}$ distal to the pylorus $\longrightarrow A I$ and $A 2$ ) and on the jejunum (5I8 cm distal to the pylorus -.$B I$ and $B 2$ ) of the Pig according to the diet $\left(A I\right.$ and $B_{I}=\operatorname{standard,A2}=$ "S.I. ", B2 $=$ maize starch) Each of the 2 daily meals (RI at 9.00 a.m., R2 at 4.30 p.m.) included $I 200$ g of flour. The height of the rectangles (irregular spiking activities) and that of the bars (regular spiking activities) is proportional to the mean height of the integration peals recorded during the phase concerned.

For each of these 4 graphs, the number of sequences per $24 \mathrm{~h}$, and the mean duration of phases $I, I I, I I I$ ave as below:
$A_{I}: n=20$
$\mathbf{I}=\mathbf{I} 7 . \mathbf{I} \div 2.0$
II $=54 . I: 2.8$
III $=3.5 \pm 0 . I$ min
Az $: n=19$
$I=25.5+3.2$
II $=48.9 \div 7.0$
III $=3.8 \pm 0 . I \mathrm{~min}$
$\mathrm{BI}: n=\mathrm{I} 5$
$I=I 8.6 \pm 2.4$
$I I=73.3$ ㄴ 5.7
III $=3.9 \pm 0 . I$ min
$\mathrm{B2}: n=\mathrm{I} 7$
$I=15.5 \pm 2.6$
II $=66.6$ 上 4.2
III $=3.2 \pm 0 . I \mathrm{~min}$ 


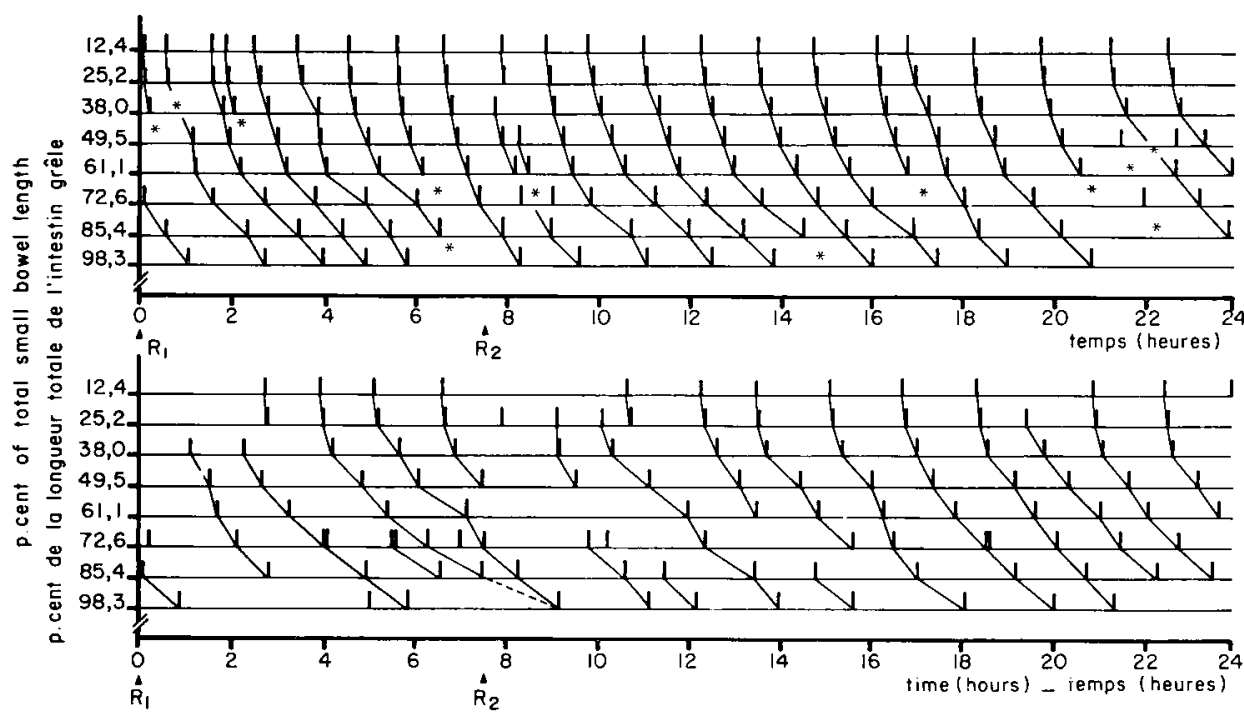

Iíg. 5. - Migration des phases d'activiti réguliève (barvettes verticales) au long de l'intestin grêle durant un cycle de $2+h$ chez un même porc : graphique supérieur : lors d'ingestion de 800 g d'aliment standard à chacun des 2 repas quotidiens (RI à 9 hoo - R2 à I6 h 30 ): graphique inférieur : lors d'ingestion de $1200 \mathrm{~g}$ du régime amidon de maïs à chacun des 2 repas quotidiens. Ces quantités représentent dans les 2 cas, compte tenu d: la dilution, une masse alimentaire identique de 2 too $\mathrm{g}$

Chaque ligne correspond à un sate d'enregistrement défui par sa distance au pylore cxprimée en pourcentage de la longueur totale de l'intestin grêle. Les astérisques soulignent les irrégularités occasionnelles de migration dans le cas du régime standard.

Migration of regular spiking activity phases (vertical bars) along the small bow'l of a pig during a $2+$ h cycle. Top-graph: intake of $800 \mathrm{~g}$ standard diet at each of the 2 daily meals (RI $=9.00$ a.m. - R2 $=4.30$ p.m.). Bottom-graph: intake of I $200 \mathrm{~g}$ maize starch dist at each of the 2 daily meals. In the 2 cases, these quantities, due to the different water dilutions of the flour, result in the same alimentary mass $(2400 \mathrm{~g})$.

Each line of graphs corresponds to a recording site, identified by its distance to the pylorus as per cent of total small bowel length. In the topgraph, the asterisks undevline the occasional migration abnormalities.

du régime standard, la quasi-totalité des complexes dénombrés sur 1'iléon terminal est effectivement le résultat de la migration de complexes depuis le duodénum proximal. Plus des trois-quarts des complexes n'atteignant pas la jonction iléocæco-colique s'effacent après avoir franchi 60 à $70 \mathrm{p}$. I 00 de la longueur totale de l'intestin grêle.

Par ailleurs, deux à trois complexes sont enregistrés simultanément sur l'ensemble de l'intestin grêle à un instant donné (fig. 6). La coïncidence entre le départ d'une activité de pointes régulière sur le duodénum et l'arrivée d'une autre activité régulière à l'iléon terminal n'est pas absolument systématique; elle est néanmoins le plus souvent observée dans une fourchette de temps de l'ordre de $\pm 20 \mathrm{mn}$.

Le calcul de la vitesse moyenne de propagation des activités régulières faisant l'objet d'une migration proximodistale complète (tabl. 4) montre que dans tous les cas cette vitesse de déplacement est la plus élevée au niveau de 1'intestin proximal. Cette particularité est liée surtout aux vitesses comprises entre 80 et $100 \mathrm{~cm}$ par mn au long du duodénum en amont de l'angle de Treitz. La décroissance est très rapide en aval de ce point. 


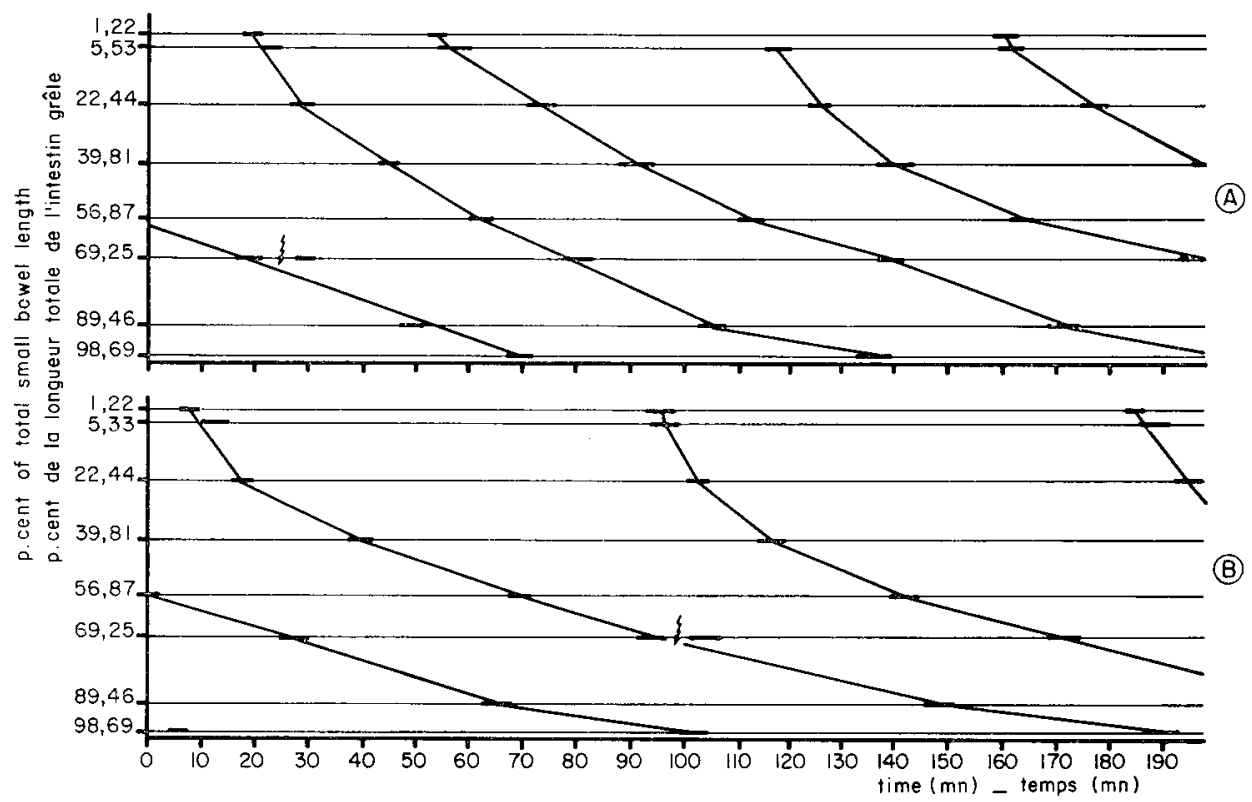

FIG. 6. - Vitesse de déplacement des bandes d'activité réguliève (épaississements des lignes) au long de l'intestin grêle du Porc selon le régime : $A=$ standard, $B=$ amidon de mä̈s. Chaque ligne correspond à un site d'enregistrement défini par sa distance au pylore exprimée en pourcentage de la longueur totale de l'intestin grêle. Les flèches brisées signalent la suspension temporaire vs. le redoublement d'une activité régulière lorsqu'une autre prend naissance en région proximale.

Velocity of propagation of the regular spiking activity phases (enlargements of lines) along the small bowel of the Pig according to the diet: $A=$ standard, $B=$ maize starch. Each line of graphs corresponds to a recording site, identified by its distance to the pylorus as per cent of total small bowel length. The broken arrows point out the temporary interruption vs. the doubling of a regular spiking activity when the following one is appearing in the proximal bowel area.

Le temps total effectivement nécessaire pour qu'une activité régulière franchisse la totalité de l'intestin grêle, mesuré chez les porcs $\mathrm{H}$ et $\mathrm{M}$, est respectivement de $122,9 \pm 4,3 \mathrm{mn}$ (22 complexes de 2 nycthémères) et de $160,5 \pm 3,3 \mathrm{mn}$ (Io complexes de 2 nycthémères).

La stabilité précédemment observée de la durée des phases d'activité régulière, quel que soit le site intestinal étudié, implique, compte tenu des vitesses de propagation constatées dans chaque cas, que la longueur du segment intestinal faisant l'objet d'une activité régulière décroît de $100-150 \mathrm{~cm}$ au niveau proximal à $50 \mathrm{~cm}$ au niveau distal. Dans un nombre de cas très limité, des " anomalies " peuvent être observées dans la migration des complexes myoélectriques. Ces phénomènes, échappant à la description générale ci-dessus consistent en : la migration d'une płase d'activité régulière depuis le duodénum proximal s'effaçant définitivement après avoir franchi seulement 20 ou $30 \mathrm{p}$. Ioo de la longueur totale de l'intestin grêle; l'apparition au niveau du jéjunum proximal, ou du jéjunum distal d'une activité régulière, non décelée en des sites plus proximaux, et migrant régulièrement en aval du site de première détection; la présence en un site d'une phase d'activité régulière qui ne s'intègre dans aucune migration proximodistale en registrée sur les autres sites d'électrodes. Ces variations restent cependant très rares chez le 
TABI,EAU 4

Tísuse moyenne de déplacement des complexes myoćlectriques au long de l'intestin grêle (ct indication de quelques valeurs moyennes individuelles) selon la nature de l'aliment et le segment intestinal considéré (exprimi selon le même mode que dans le tabl. 3)

Hean velocity of the myoelectric migrating complexes (M.M.C.) along the small bovel (and indication of some mean individual values) according to the diet and to the small bowel area considered (the limits of which are expressed as in table 3)

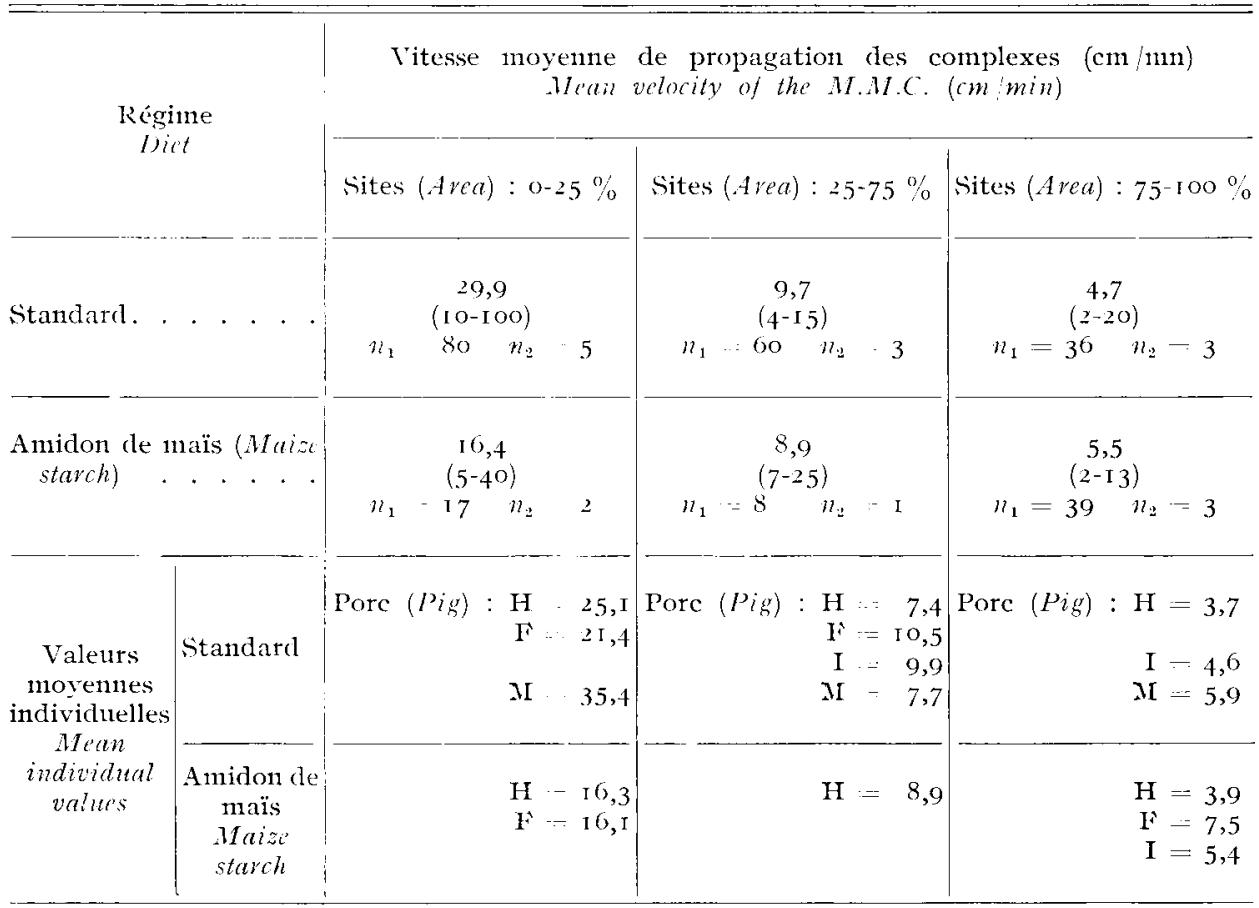

$\eta_{1}$ : Nombre de complexes propagés sur la totalité de l'intestin grêle (Number of MMC propagating along the whole small boiel).

th. : Nombre de pores (Numbir of pigs).

$($ ) : Valeurs extrimes (Komge).

porc consommant le régime standard. Par contre, un autre type de particularité beaucoup plus fréquent consiste en la suspension temporaire ou le redoublement d'une phase d'activité régulière passant au niveau des sites 60 à $70 \mathrm{p}$. Ioo de la longueur intestinale lorsque prend naissance au niveau duodénal la phase d'activité régulière consécutive (fig. 6). Ce phénomène est totalement distinct de 1'interruption transitoire d'une période d'activité régulière par la propagation rapide d'une onde péristaltique (fig. 7) comme cela est fréquemment observé au niveau de l'iléon. Cette interruption correspond au silence électrique habituellement consécutif à ce type d'activité rapide très intense, quel que soit l'électromyogramme environnant. 


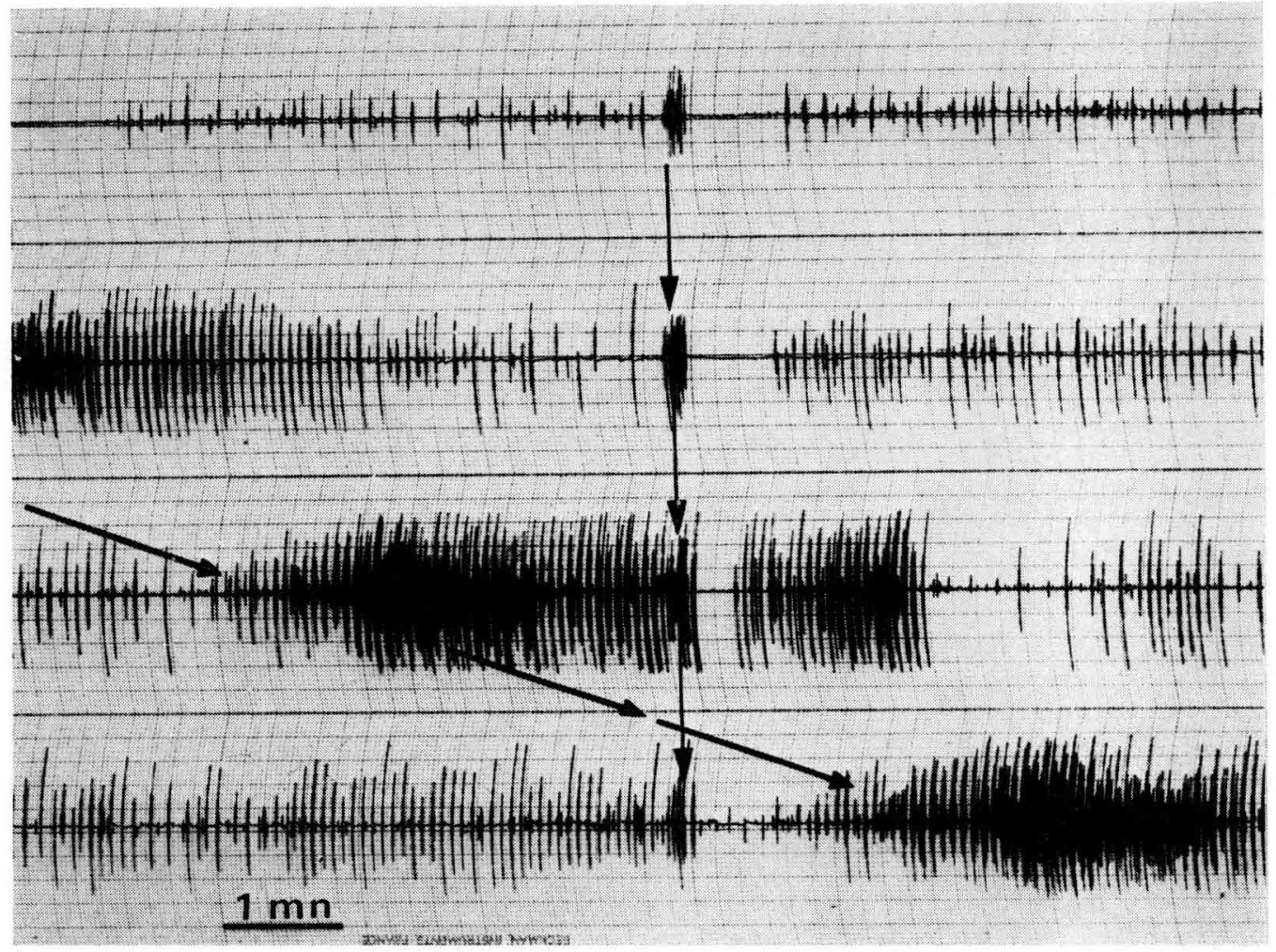

FIG. 7. - Electromyogramme ilóal en 4 sites espacis de $5 \mathrm{~cm}$ dont le plus distal (tracé inférieur) se trouve à $3 \mathrm{I} \mathrm{cm}$ de la jonction iléo-caeco-colique chez le Porc. Noter la différence de vitesse entre la propagation rapide d'une onde péristaltique et la lente migration d'une activité régulic̀re. L'onde péristaltique franchit la bande d'activité régulière. Le silence électromyographique consécutif à l'onde péristaltique est observable quelle que soit l'activité électrique préalable à son passage; il en résulte une interruption transitoive de l'activité de pointes régulière.

Electromyographic activities in 4 ileal sites, each one $5 \mathrm{~cm}$ apart from another, the most distal of them (bottom graph) is $31 \mathrm{~cm}$ above the ileo-caeco-colic junction in a Pig. Note the fast propagation of a peristaltic wave and the slow migration of a regular spiking activity. The electromyographic pause consecutive to the peristaltic wave may be observed whatever the surrounding electromyogram. It results in a transient interruption of the regular spiking activity.

2. - Infuences de l'alimentation, d'ordre qualitatif et chronologique

A. - Effets d'un régime semi-synthétique à base d'amidon de maïs 2 repas par jour)

Comme le montre le tableau $I$, les animaux ayant reçu le régime amidon de maïs (ou S.F.) ont été préalablement testés avec le régime standard. Le sens des effets observés chez chacun d'entre eux étant analogue, chaque porc étant son propre témoin, l'expression de données moyennes est possible pour ce régime comme pour le régime standard, a fin d'échapper à l'importance, signalée précédemment, des variations entre individus. 


\section{a) Périodicité et durée des phénomènes}

Les enregistrements réalisés chez les porcs accoutumés à la consommation 2 fois par jour des aliments semi-synthétiques amidon de maïs ou "S.F. " permettent de retrouver dans cette situation des complexes myoélectriques répondant à la description générale précédemment fournie dans le cas d'ingestion d'un régime standard.

Le nombre moyen de complexes (séquence I + II + III) enregistrés en $24 \mathrm{~h}$ au niveau du quart proximal de l'intestin grêle est systématiquement plus faible (tabl. 3) qu'avec le régime standard. Ce nombre est en moyenne du même ordre de grandeur pour les aliments amidon de maïs et "S.F. ". Cependant la réduction du nombre de complexes, observée lorsque leur dénombrement par 24 h est effectué sur des sites de plus en plus distaux, est plus faible pour l'amidon de maîs (25 p. I oo - estimation globale sur la base des moyennes) que pour l'aliment standard. Ce fait conduit à enregistrer en moyenne au niveau iléal un plus grand nombre de complexes en $24 \mathrm{~h}$ avec l'amidon de maïs.

Les intervalles séparant le début de 2 phases consécutives d'activité de pointes régulière (i.e. la péricde des complexes) restent au niveau des $75 \mathrm{p}$. Ioo proximaux de l'intestin grêle (fig. 4) du même ordre de grandeur chez les porcs consommant l'amidon de maïs que chez ceux recevant 1'aliment standard. Par contre, le plus grand nombre de complexes migrant au niveau distal par $24 \mathrm{~h}$ en régime amidon de maïs conduit à observer en ce site un délai de récurrence de 90 à Ioo mn, c'est àdire plus court que lors d'ingestion de l'aliment standard.

L'analyse de la décomposition de ces périodes en leur différentes phases montre que la durée (tab1. 3) des activités de pointes régulières (III) n'est pas affectée par la composition du régime quel que soit le site intestinal considéré. Alors qu'un allongement de la durée des activités irrégulières est constaté en régime standard. pour les sites les plus distaux, ces phases II ont en régime amidon de maîs une durée analogue quel que soit le site intestinal concerné. Il faut, en outre, souligner que l'intensité des activités rapides iléales au cours des phases II est beaucoup plus faible en régime amidon de maïs qu'en régime standard (fig. 8).

En définitive, l'allongement global de la durée des complexes entre le duodénum et l'iléon, de l'ordre de $20 \mathrm{mn}$ seulement en régime amidon de maïs (contre 40 en régime standard), est supporté en totalité par la prolongation de la période de quiescence (I).

\section{de mais}

b) Migration des complexes myoélectriques chez le porc recevant le régime amidon

L'enregistrement simultané des électromyogrammes en divers sites régulièrement échelonnés tout au long de l'intestin grêle montre (fig. 5) que, dans le cas du régime amidon de maïs, seulement la moitié environ des phases d'activité régulière enregistrées au niveau iléal sont l'aboutissement d'une migration au long de la totalité de l'intestin grêle. D'une façon générale, la migration des complexes est pour ce régime beaucoup plus anarchique, en particulier au cours de la période diurne comportant les distributions alimentaires. Cet aspect global résulte d'une fréquence beaucoup plus élevée de manifestation, en régime amidon de maïs, de ce qui a été décrit comme des anomalies occasionnelles de migration en régime standard.

Indépendamment de cette fréquente discontinuité dans la migration des activités régulières, il apparaît aussi que leur vitesse moyenne de déplacement au long de l'intestin grêle entier est, chez les pores consommant l'aliment amidon 


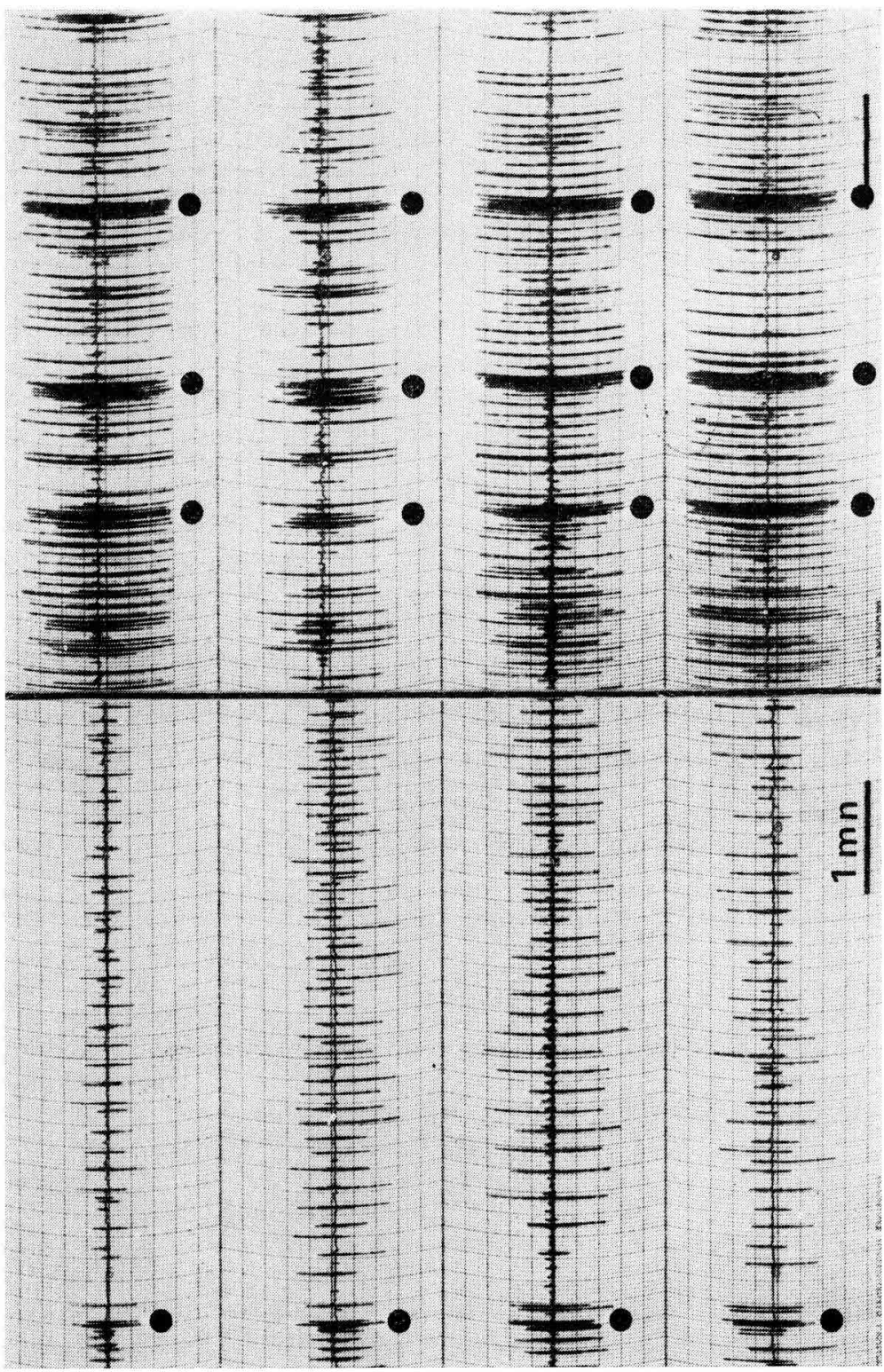

FIG. 8. - Modification de l'intensité des activités rapides irrégulières selon la nature du régime chez le Porc. Électronyogrammes dérivés sur l'iléon en 4 sites espacés de $5 \mathrm{~cm}$ dont le plus distal (tracé inférieur) est situé à $3 I \mathrm{~cm}$ de la jonction iléo-caeco-colique. A gauche : végime amidon de mä̈s; à droite : régime standard. Noter dans ce dernier cas la puissance et la fréquence des ondes péristaltiques rapides (soulignées par les points). Dans les 2 cas, l'enregistrement est réalisé $8 \mathrm{mn}$ avant l'apparition de l'activité régulière.

Modifications in the intensity of the irregular spiking activities according to the diet in the Pig. Electromyograms recorded in 4 ileal sites, each one $5 \mathrm{~cm}$ apart from another, the most distal of them (bottom graph) is $31 \mathrm{~cm}$ above the ileo-caeco-colic junction. On the left: maize starch diet; on the right: standard diet. The fast propagated peristaltic waves (dots) are very strong and numerous with the standard diet. The 2 recordings were done exactly 8 min before the appearance of a regular spiking activity. 


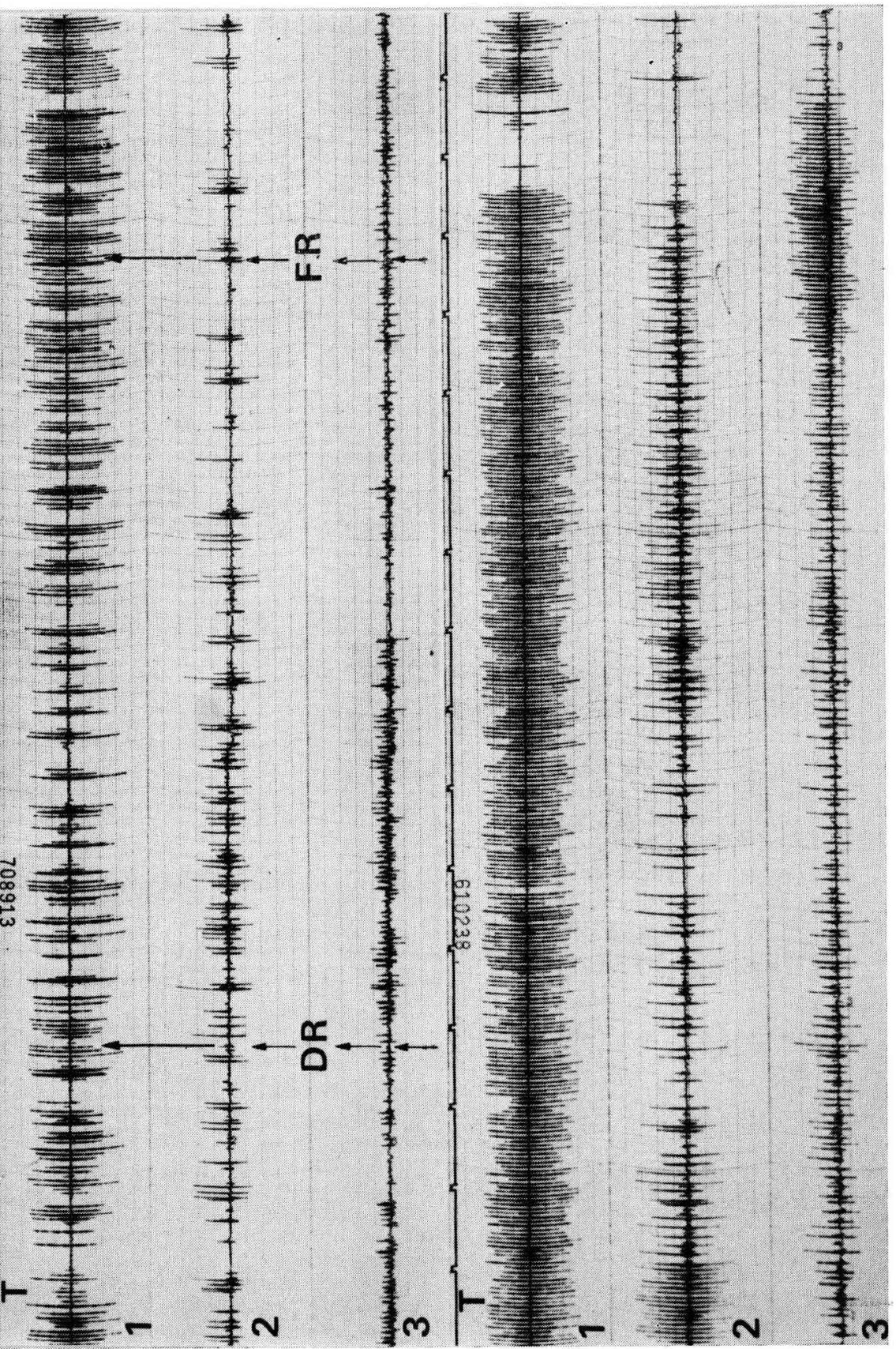

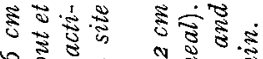

어요

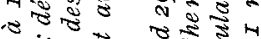

$\vec{\Sigma}$ जे

ॠू

पू है है

ปूस

0.0 I

5.

2.

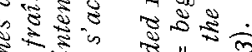

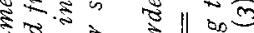

है है ङ

है०

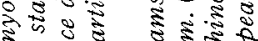

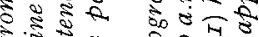

要糙

I)

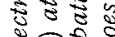

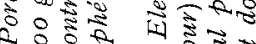

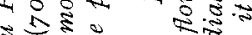

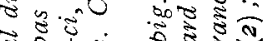

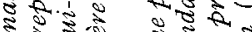

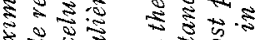

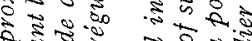

จ

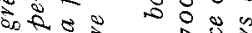

₹

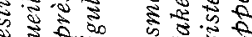

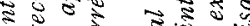

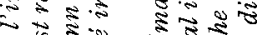

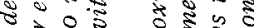

ह

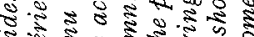

है:

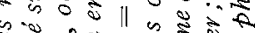

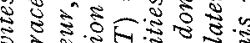

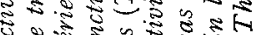

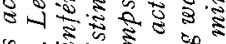

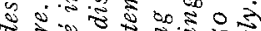

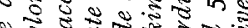

डै

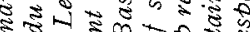

काष 50

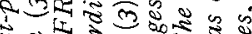

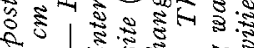

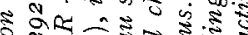

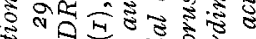

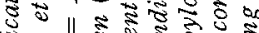

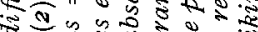

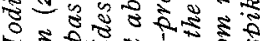

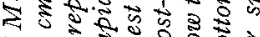

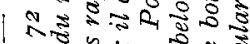

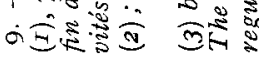
它 


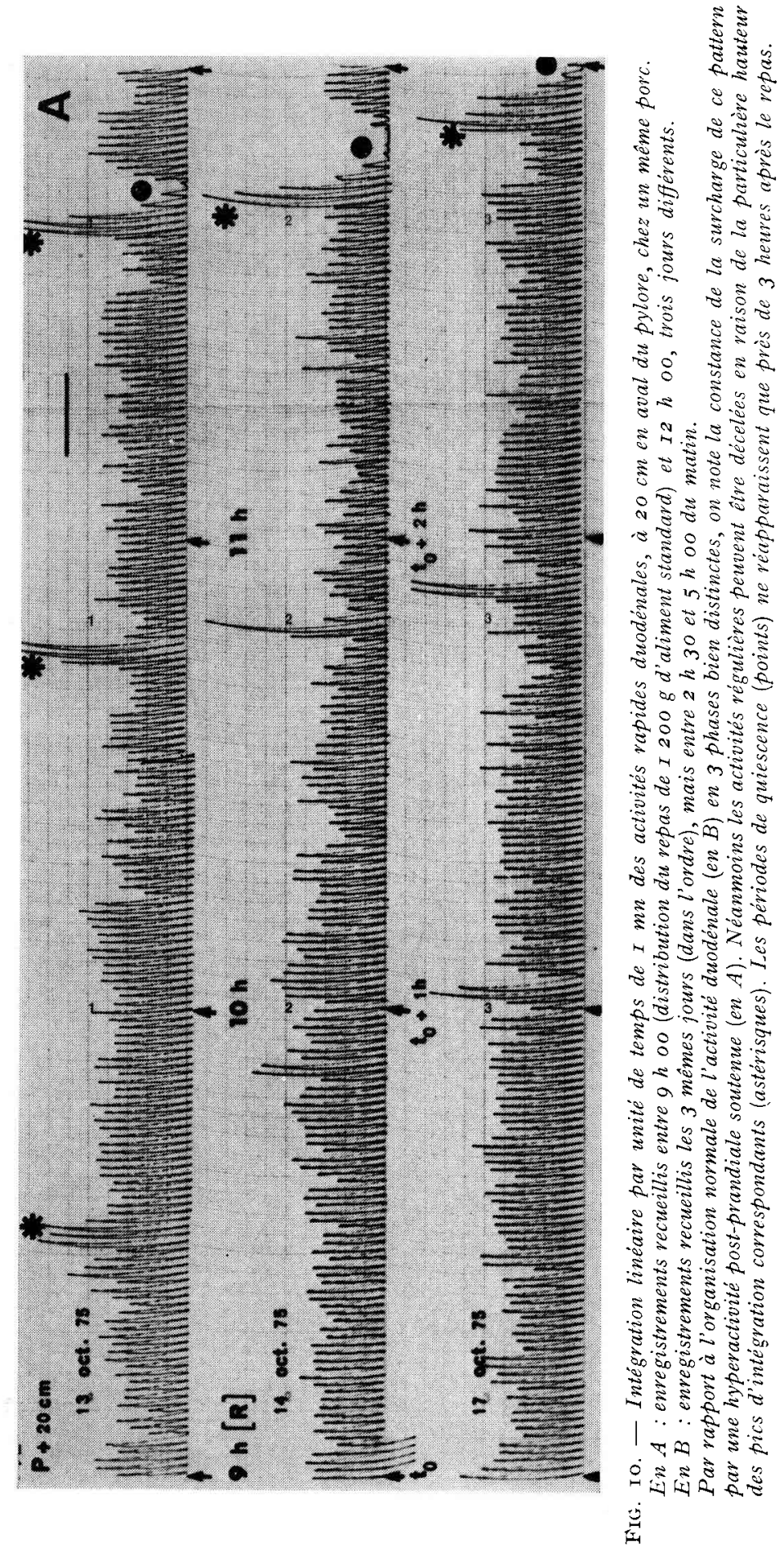




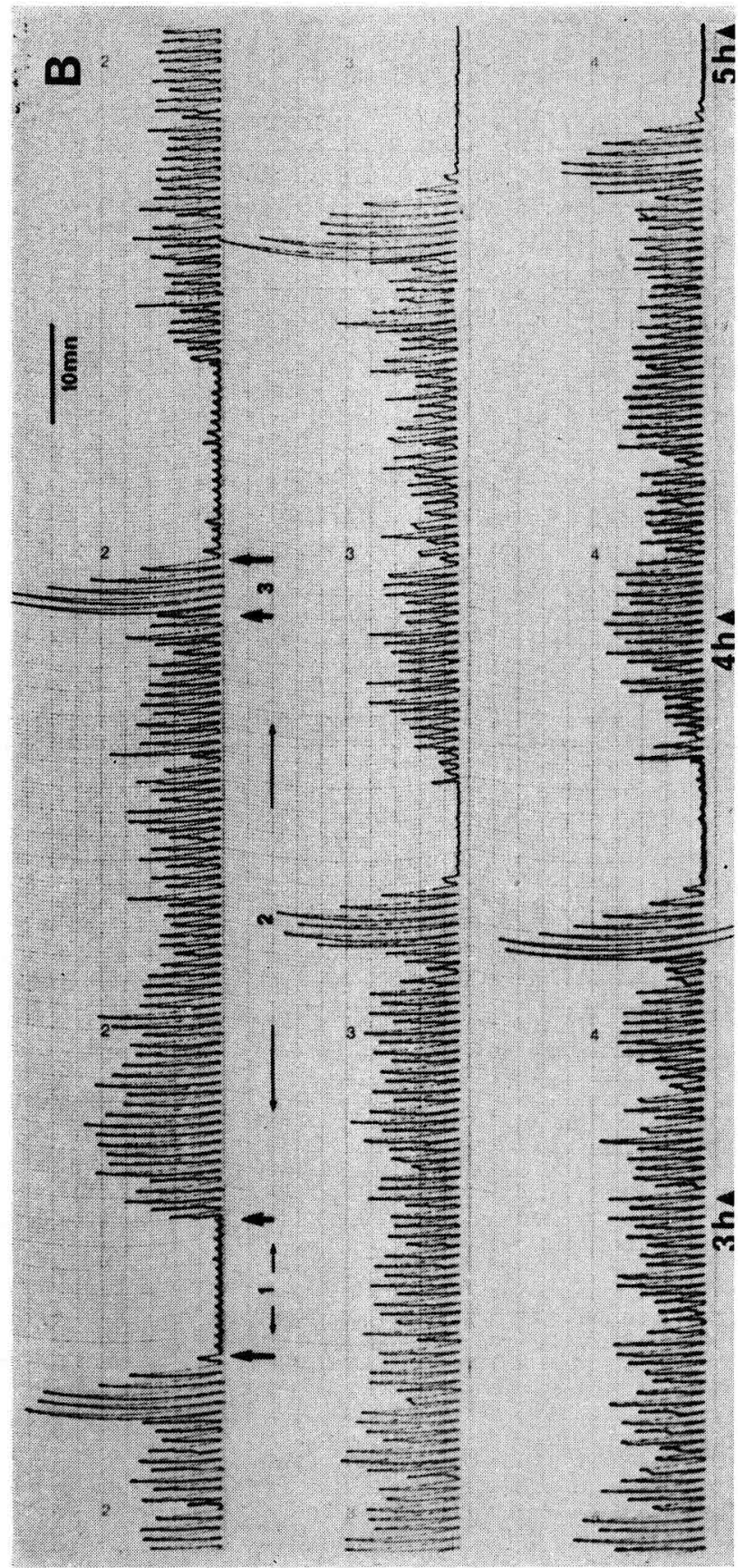

章章

$\overrightarrow{\mathrm{E}}$

起方

른

is

is

ఫ

离

के है

का का

इ

Do

म. 2

ङ

क 8

क

저 $\approx$

ऊ हु

केष्ट

2. 0 m

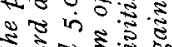

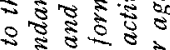

द क वे

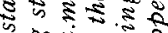

के

8 :

क व

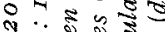

คी

₹

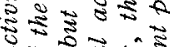

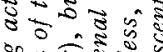

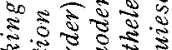

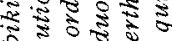

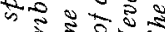

के है द

क्षे क्षे

छ के $\dot{0} \dot{0}$

竎

\& 5 क 50

ङ

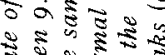

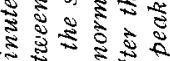

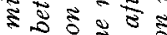

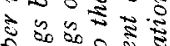

표요

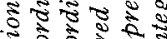

उ के है है से

के 2 है की

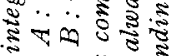

हैइ हूँ 
de maïs, inférieure de $33 \mathrm{p}$. Ioo à celle mesurée en régime standard. Cette diminution de la vitesse de déplacement des activités régulières, liée au régime (fig. 6), tient pour l'essentiel à une vitesse plus faible d'environ $45 \mathrm{p}$. Ioo au long du quart proximal de l'intestin grêle (tabl. 4). Pour ce territoire, le régime "S.F." conduit à des valeurs encore plus faibles ( $3,2 \mathrm{~cm}$ par $\mathrm{mn}-n_{1}=26, n_{2}=2$ ) que celles enregistrées pour 1'aliment amidon de maïs. Il résulte de ces différences de vitesse un allongement en régime amidon de maïs par rapport au régime standard, d'environ $60 \mathrm{mn}$. du temps théoriquement nécessaire an franchissement de tout 1'intestin grêle par une activité régulière. Par exemple, les valeurs moyennes effectivement enregistrées dans le cas du Porc $\mathrm{H}$ (fig. 6) sont respectivement de $122,9 \pm 4,3 \mathrm{mn}$ (22 complexes de 2 nycthémères en régime standard) et de $\mathrm{I}_{3}, \mathrm{I} \pm 4,2 \mathrm{mn}$ ( $\mathrm{I} 3$ complexes sur 2 nycthémères en régime amidon de maïs) soit un écart de $40 \mathrm{mn}$ des temps réels de franchissement de la totalité de l'intestin grêle.

\section{B. - Influence de la chronologie d'ingestion des repas}

\section{a) Distribution ad libitum vs. 2 repas en régime standard}

L'ingestion d'aliment standard à l'occasion de 2 repas quotidiens imposés est responsable au niveau de l'intestin grêle proximal, d'une augmentation de l'activité irrégulière (fig. 9) qui se manifeste à la fois dans une fréquence des bouffées de pointes fortement accrue, une amplitude plus grande des potentiels d'action qui les composent, et un allongement de la durée de cette phase II des complexes. Cette intensification globale est telle que la phase III d'activité régulière ne peut souvent plus être différenciée, à l'examen direct des enregistrements, dans une activité rapide quasi continue durant environ $2 \mathrm{~h}$, et que la phase I de silence peut être totalement abolie. Néanmoins, l'activité de pointes régulière peut être dans la quasi totalité des cas identifiée par le biais de l'intégration des activités rapides, en raison d'une plus grande hauteur des pics d'intégration exprimant 1'activité intestinale par unité de temps (fig. IO). Chez nos porcs, pout l'ingestion usuelle d'un repas d'aliment standard de $\mathrm{I} 200 \mathrm{~g}$ de farine, soit une masse alimentaire de $3600 \mathrm{~g}$ après dilution, consommée en $8 \mathrm{mn}$ en moyenne, les activités régulières redeviennent aisément identifiables, et les phases de silence réapparaissent systématiquement au-delà de $\mathrm{I} 20$ à I5O mn après le début du repas du matin (fig. Io) et de 60 à 90 mn après le début du second repas (à $\mathrm{I} 6$ h 30 ).

Pour ce mêne mode de distribution (2 repas) du même aliment standard, l'augmentation de la quantité ingérée (entre 600 et I $200 \mathrm{~g}$ par repas) se traduit, au niveau proximal (20 p. Ioo de la longueur de l'intestin grêle), par l'intensité moyenne accrue des phases d'activité irrégulière et la prolongation de 1'hyperactivité postprandiale. Par ailleurs, seul l'iléon terminal manifeste une nette intensification des activités dispersées durant 30 à $60 \mathrm{mn}$ après le repas du matin. Ce phénomène reste néanmoins sans commune mesure avec l'activité postprandiale duodénale.

Par rapport à cette situation ( 2 repas), on constate que 1'hyperactivité post prandiale n'est pas observable à aucun moment du nycthémère chez le Porc ingérant spontanément ad libitum 3200 à $3700 \mathrm{~g}$ d'aliment standard granulé au cours des $24 \mathrm{~h}$. Une telle comparaison n'a pas été effectuée en régime amidon de maïs. Dans ce dernier cas et en situation de 2 repas/jour, 1'hyperactivité post-prandiale est observée durant $3 \mathrm{~h}$ au plus après ingestion de I $200 \mathrm{~g}$ à $9 \mathrm{~h}$. 
b) Organisation comparée des activités intestinales au cours des périodes diurne et nocturne chez le porc recevant 2 repas

La comparaison de l'organisation des activités intestinales au cours de la période "diurne " ( $9 \mathrm{~h}$ à $2 \mathrm{I} h$ ) et de la période "nocturne " (2I h à $9 \mathrm{~h})$ permet de constater un allongement constant d'environ to mn de la durée des phases de quiescence au cours de la période nocturne (tab1. 5). La durée des activités régulières ne varie pas. Celle des activités irrégulières n'est pas significativement abrégée en période nocturne.

Globalement, la durée moyenne des complexes (I + II + III) enregistrés au cours de la période nocturne tend à être plus longue que celle des complexes enregistrés en période diurne (tabl. 6). Cette variation n'est cependant pas systématique.

L'analyse comparative des vitesses de propagation respectives des complexes enregistrés en période diurne et nocturne fait apparaître, en moyenne générale, une tendance au ralentissement de la migration des phases d'activité régulière durant la nuit. Cependant une évolution inverse est parfois enregistrée au niveau du quart proximal de l'intestin grêle, en particulier avec le régime amidon de maïs.

Enfin, on note que les variations de 1'intensité relative des activités régulières ne peuvent être reliées à un facteur contrôlable; l'intensité des phases d'activité irrégulière n'est pas systématiquement réduite durant la période nocturne.

\section{Discussion}

Ainsi qu'il a été antérieurement décrit (LAPLACE et GERMAIN, I976; RUCKEBUSCH et BUENo, I976) le Porc présente une organisation des activités rapides de l'intestin grêle sous forme de complexes myoélectriques, de récurrence cyclique, migrant lentement au long de l'organe. Il répond donc à la description générale admiise à ce jour pour plusieurs espèces animales.

\section{I. - Les complexes myoélectriques chez le Porc par rapport à d'autres espèces}

\section{a) De la permanence des complexes}

La place du Porc dans une classification fonctionnelle des principales espèces étudiées sur ce plan, peut être définie comme strit:

- Chez le Chien, carnivore à intestin grêle court (six fois la longueur du corps selon BARONE, I976), les complexes myoélectriques sont absents chez l'animal alimenté (CODE et MarLetT, I975). Il en est de même chez le Rat (RuCKËBusch et FERRE, I973) ou 1'Homme (HELLEMANS et al., I977), omnivores à aptitudes carnivores certaines, dotés d'un intestin grêle de longueur également modérée relativement à la longueur du corps (dix fois chez l'Homme selon BARONE, I976).

- Le Porc, omnivore consommant une nourriture composée dans sa plus grande partie de constituants d'origine végétale, et doté d'un intestin grêle sensiblement plus long (treize fois la longueur du corps; BARONE, 1976), présente des complexes myoélectriques, qu'il soit à jeun ou alimenté ad libitum, et ce tant chez le Porc miniature (Ruckebusch et Bueno, I976) que chez nos Pores Large White. Cependant, lors de distribution de repas limités, cette organisation habituelle cède la place au niveau du seul intestin grêle proximal, et pour un temps limité (I20 mn en moyenne) à une activité de pointes quasi continue. 


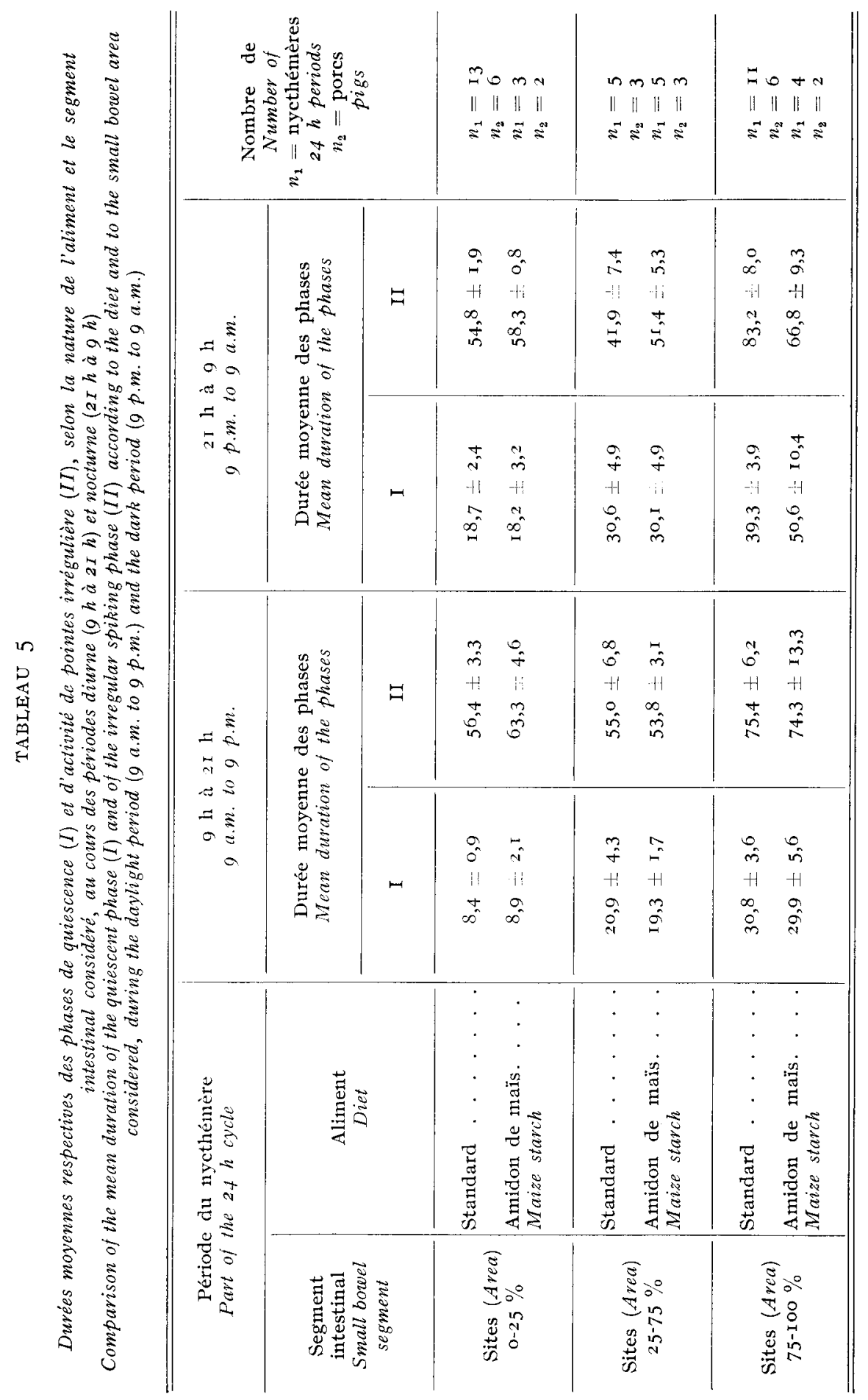




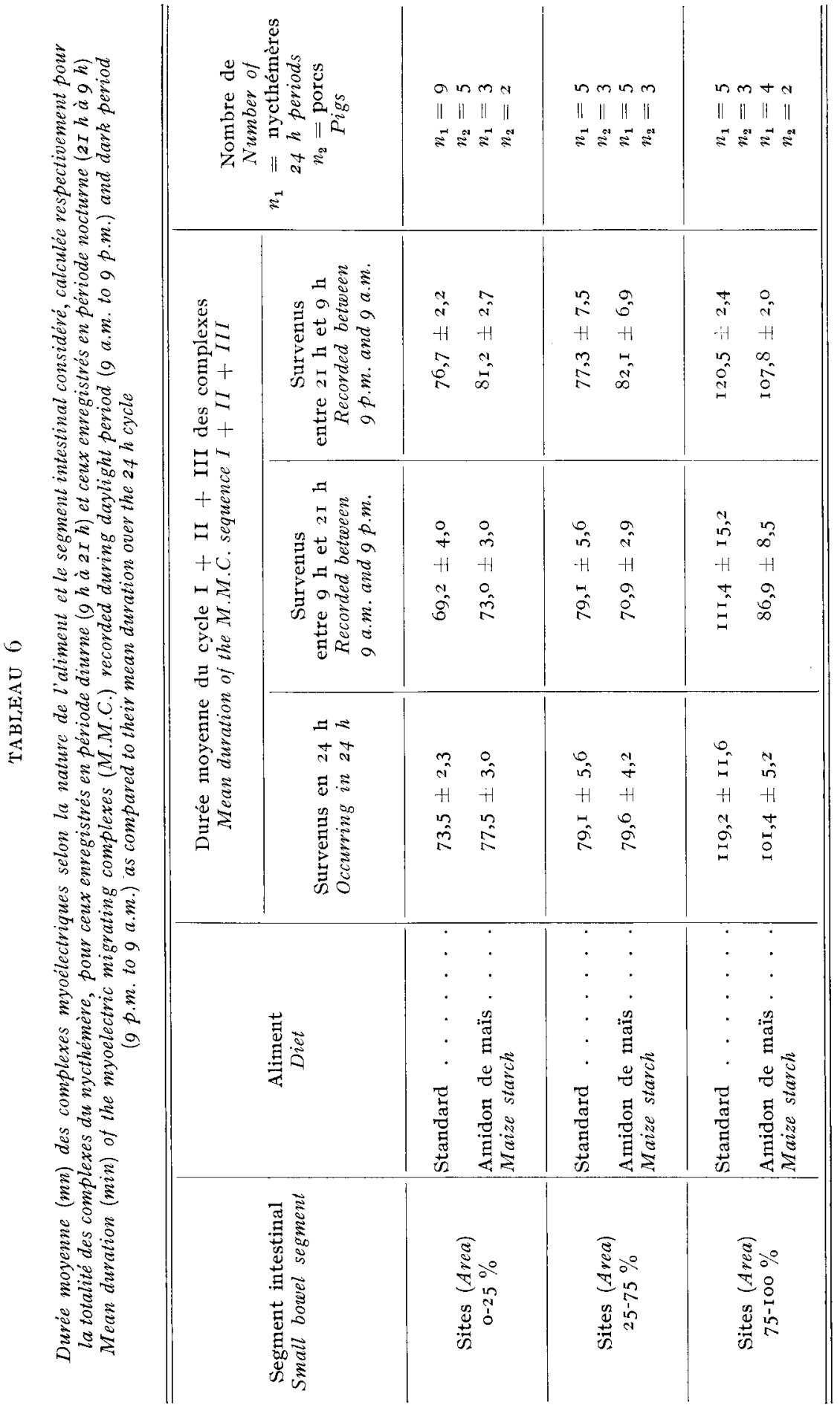


- Le Mouton, herbivore polygastrique, ou le Lapin, herbivore monogastrique, sont des espèces à intestin grêle relativement important ( I 2 fois la longueur corporelle chez le Lapin) ou très long ( 25 fois la longueur du corps chez les petits ruminants, BARONE, I 976). Les complexes myoélectriques sont présents en permanence chez l'un comme chez l'autre en situation d'alimentation ad libitum (GRIVEL et RuCKEBUSCH, I972).

En somme, pour cet aspect particulier de sa physiologie digestive, le Porc semble globalement plus proche d'animaux herbivores, tels que Mouton et Lapin, que d'autres espèces omnivores (Rat, Homme) ou carnivores (Chien).

\section{b) Particularités des complexes myoélectriques chez le Porc}

Indépendamment de ces aspects interspécifiques, 1'absence des complexes myoélectriques n'a jamais été observée chez les Porcs utilisés dans ce travail. Leur absence a été signalée chez 3 chiens sur 7 par CARLSON, BEDI et CODE (I972) et chez I chien par SzURSzEWSKI (I 969). Selon ce dernier auteur, cette anomalie semble pouvoir être reliée à un état pathologique associé qui a conduit 1'animal à la mort. Cette observation est à rapprocher de celle de DARDI_LAT (I975) d'une désorganisation du profil moteur de l'intestin grêle comme premier signe, chez le Veau, de phénomènes pathologiques non nécessairement digestifs.

I, a relation entre les activités rapides antrales et duodénales, observée par Ruckebusch et Bunno (I976) chez les Pores miniatures, est ici confirmée (suspension des activités antrales lors du départ d'une activité régulière sur le duodénum). Cette relation particulière est susceptible de rendre compte pour partie des irrégularités post prandiales du débit gastro-duodénal tel qu'il a été antérieurement décrit (LAPLACE et Tomassone, I970).

Le nombre de séquences complètes enregistrées par 24 h en un même site chez nos Porcs révèle une variabilité importante entre individus, alors que ce même dénombrement fournit des valeurs relativement stables pour un même site intestinal d'un jour à l'autre chez un même Porc. Ceci a pour conséquence au plan méthodologique, que l'étude d'une influence telle que celle du régime, doit être effectuée en utilisant chaque animal comme son propre témoin. Seule 1'homogénéité de nature et de sens des effets observés autorise le calcul de valeurs moyennes destinées à simplifier la description générale. Par ailleurs, cette variabilité constatée entre nos porcs concorde avec les données de CODE et MARLETT (r975) relatives au Chien, révélant une variabilité beaucoup plus grande de la durée moyenne des cycles entre chiens qu'entre jours d'enregistrement. Il y a également concordance en ce qui concerne l'allongement de la durée moyenne des complexes dans le sens oral-aboral entre le Chien (CODE et MARLETT, I975) et le Porc.

Cette plus grande durée des séquences enregistrées au niveau iléal, i.e. leur nombre plus réduit en $24 \mathrm{~h}$ par rapport à ce qui est observé pour le duodénum, correspond chez nos Porcs, à un pourcentage moyen de complexes ne franchissant pas la totalité de l'intestin grêle, de l'ordre de $40 \mathrm{p}$. Ioo. Cette valeur peut être rapprochée des valeurs signalées pour d'autres espèces : 8I p. Ioo des complexes (SzURszewski, r969) ou les deux tiers d'entre eux (GRIVEL et RUCKEBUSCH, I972) seulement migrent jusqu'à l'iléon chez le chien à jeun. Il en est de même chez le Mouton et le Lapin, pour lesquels les complexes qui disparaissent ne franchissent en général que $40 \mathrm{p}$. Ioo de la longueur de l'intestin contre $60 \mathrm{p}$. Ioo (Grivel et Ruckebusch, I 972) ou 75 p. Ioo (SzUrszewski, I969) chez le Chien. Sur ce point particulier, il y a analogie entre le Chien et le Porc puisque, chez nos animaux, les complexes s'effacent éventuellement après avoir franchi quelques 60 à $70 \mathrm{p}$. Ioo 
de la longueur de l'intestin grêle. A cela, on peut ajouter que le Porc n'est pas seul à présenter diverses anomalies occasionnelles de la migration des complexes, puisque SzURSzEWSKI (I969) décrit la naissance de complexes au niveau duodénal ou jéjunal proximal, et que CODE et MARLE'T'T (1975) signalent l'absence possible de la phase d'activité régulière au niveau du tiers proximal, ou au niveau du tiers distal de 1'intestin grêle du Chien.

En ce qui concerne la durée moyenne des 3 phases constitutives des complexes myoélectriques, celle des activités régulières s'avère stable au long de l'intestin grêle du Porc, alors qu'elle décroît chez le Chien, et augmente chez le Mouton et le Lapin dans le sens oral-aboral (GRIVEI, et RUCKEBUSCh, I972). Code et MarLETT (I 975) ne relèvent pas de différence systématique de la durée des phases d'activité irrégulière entre sites d'enregistrement. Par contre, on note chez le Porc consommant un régime standard que l'activité irrégulière, qui représente en moyenne 73 p. roo de la durée du cycle pour le quart proximal de l'intestin grêle, en constitue $6 \mathrm{I} \mathrm{p}$. Ioo pour les $50 \mathrm{p}$. Ioo médians du viscère, mais $69 \mathrm{p}$. Ioo au niveau de son quart distal. Enfin, un allongement systématique de la période de quiescence est observé dans le sens oral-aboral, concomitant de l'allongement de la durée moyenne des cycles, ou de la réduction du nombre de séquences complètes en $24 \mathrm{~h}$ selon le même gradient.

\section{c) Migration des complexes myoélectriques}

Si l'on fait abstraction des migrations incomplètes qui rendent compte de la variation proximo-distale du nombre de complexes enregistrés en chaque site, la stabilité de la répétition et de la migration des complexes myoélectriques paraît analogue chez le Porc (en régime standard) et chez le Chien à jeun. Dans cette espèce, en effet, SzURszEwSKI (I969) ainsi que CODE et MARLETT (I975) constatent que lorsqu'une activité régulière atteint l'iléon, une autre prend naissance au niveau proximal, ou est sur le point de se développer. Ceci implique qu'existent toujours sur l'intestin grêle du Chien à jeun au moins un et au plus deux complexes myoélectriques; CoDE et MARLETT (I975) ajoutent encore que, en un site donné, la phase d'activité régulière survient à peu d'écart près $(25 \mathrm{mn})$ à la même heure du nycthémère. Chez nos Porcs, si ce dernier fait ne peut être retrouvé de façon systématique, la coïncidence entre la naissance d'une activité régulière sur le duodénum et l'arrivée d'une autre phase homologue à l'iléon terminal est observable à $\pm 20 \mathrm{mn}$ près. Mais il ne s'agit pas chez le Porc de deux séquences consécutives. Ce sont en effet, deux ou trois complexes qui sont à tout instant présents en quelque point de l'intestin grêle du Porc, tout comme chez le Veau (Dardiliat et Marrero, I977).

En ce qui concerne la vitesse de migration des complexes myoélectriques, le Porc s'avère beaucoup plus proche des Herbivores que des Carnivores. En effet, la vitesse moyenne calculée au niveau du quart proximal de l'intestin grêle (environ $30 \mathrm{~cm} / \mathrm{mn}$ ) est intermédiaire entre les valeurs indiquées par GRIVEL et RUCKEBUSCH (I972) pour les Io p. Ioo proximaux de l'intestin grêle du Lapin (Io cm / mn) et $\mathrm{du}$ Mouton (I00 $\mathrm{cm} / \mathrm{mn}$ ). Cette vitesse moyenne est très supérieure à celle enregistrée pour le même territoire chez le Chien $(3,5$ à I I, $7 \mathrm{~cm} / \mathrm{mn}$ selon les auteurs; Szurszewki, I969; Carlson, Bedi et Code, I972; Grivel, et RuCkEbusch, I972; CODE et MARLETT, I975). Elle est, au niveau de l'iléon du Porc, supérieute $(4,7 \mathrm{~cm} /$ mn en moyenne) à celle enregistrée chez le Chien $(0,9$ à $2,5 \mathrm{~cm} / \mathrm{mn}$ selon les mêmes auteurs), analogue à celle apportée pour le Lapin $(3,4$ à 4,2 $\mathrm{cm} / \mathrm{mn}$; GRIVEL et Ruckebusch, I972), et inférieure à celle mesurée chez le Mouton (30 à $43 \mathrm{~cm} / \mathrm{mn}$; Griver, et RUCKEBUSCH, I972). 
Ces caractéristiques, établies en régime standard pour nos Porcs, conduisent à un temps de franchissement de l'intestin grêle total d'environ I20 mn, soit du même ordre que chez le Chien (97 à I20 mn), le Lapin (86 à I07 mn) ou le Mouton (ro3 à I Io mn). Cette similitude étend donc au Porc le fait, établi pour ces 3 dernières espèces par GRIVEL et RUCKeBUSCH (I972), selon lequel il existerait une relation entre la vitesse de migration des activités régulières et la longueur de 1'intestin grêle. Ceci conduit à un temps de franchissement du viscère du même ordre de grandeur dans tous les cas en dépit d'une différence de longueur de l'ordre de I à Io.

\section{2. - Infuences respectives de la réplétion digestive et de la composition du régime}

Parmi les facteurs potentiels de variation des complexes myoélectriques, les changements du degré de réplétion digestive et de la composition de l'aliment paraissent devoir être retenus sur la base des résultats recueillis. Leur dissociation n'est cependant pas toujours aisée. Ainsi, la fréquence des migrations incomplètes est relativement accrue chez le Porc au cours des heures diurnes postprandiales, ce qui peut témoigner d'une certaine influence de la réplétion digestive. Mais surtout elle est beaucoup plus importante dans le cas du régime amidon de maîs que dans le cas du régime standard, à ingéré égal de matière sèche ou à masse alimentaire ingérée égale (par exemple $2400 \mathrm{~g}$ de soupe représentent $800 \mathrm{~g}$ d'aliment standard en dilution $\mathrm{I} / 2$ et $\mathrm{I} 200 \mathrm{~g}$ d'alimient amidon de maïs en dilution $\mathrm{I} / \mathrm{I}$ ). De fait, il n'existe pas de corrélation entre la quantité de matière sèche ingérée et le volume de digesta passant dans le duodénum en $7 \mathrm{~h}$ ap rès le repas, alors que ce même volume est corrélé avec le volume total de la ration ingérée, compte tenu de la dilution préalable de l'aliment et quelle qu'en soit la composition (BorGIDA et LAPLACE, I977). L'influence de la réplétion semble hors de cause puisqu'identique pour les 2 régimes, par exemple dans le cas illustré par la figure 5 . Il paraît donc fort probable que l'irrégularité de la migration des activités régulières, observée pour l'aliment amidon de maïs, soit une conséquence de la composition de cet aliment, i.e. de la quantité relative de certains des nutriments apportés.

\section{a) Variations liées à la réplétion digestive}

\section{Alimentation ad libitum}

RUCKEBUSCH et BUENO (I976) enregistrent en moyenne I 8 complexes par $24 \mathrm{~h}$ chez le Porc, qu'il soit laissé à jeun durant $24 \mathrm{~h}$ ou alimenté ad libitum. Cette valeur est plus élevée que lors de distribution double (I6) ou unique (I3). Dans le cas du Porc $\mathrm{H}$, seul soumis à une distribution ad libitum, nous avons enregistré une diminution du nombre de complexes par $24 \mathrm{~h}$. Mais cet animal présentait habituellement un nombre relativement élevé de complexes au niveau iléal. Aussi, cette réduction, de $I_{5}$ complexes $/ 24 \mathrm{~h}$ pour une double distribution quotidienne à I2 en situation ad libitum, ne peut être tenue pour une contradiction. Néanmoins, si l'ingéré est analogue dans les situations de double distribution quotidienne et de repas quotidien unique (30 à $40 \mathrm{~g}$ de matière sèche $/ \mathrm{kg}$ de poids vif) selon RUCKBUSCH et BUENo (I976), ces auteurs n'indiquent pas l'ingéré en situation d'ad libitum. Or notre Porc $\mathrm{H}$ a ingéré spontanément en moyenne I kg d'aliment sec de plus $(3400 \mathrm{~g} / 24 \mathrm{~h}$ ) dans cette même situation que lors de distribution de 2 repas 
$(2400 \mathrm{~g} / 24 \mathrm{~h})$. Il pourrait donc y avoir ici une influence de la réplétion digestive, conduisant par exemple à accroître la fréquence des migrations incomplètes au niveau du jéjuno-iléon.

\section{Différences diurne/nocturne lors de double distribution}

Indépendamment de cet aspect, la distribution des 2 repas quotidiens à heures fixes ( 9 h et I6 h 30) conduit chez nos Porcs à une différence importante du degré de réplétion de l'intestin grêle pendant les heures diurnes et les heures nocturnes. L'analyse comparative des caractéristiques des complexes au cours des $\mathrm{I} 2 \mathrm{~h}$ comportant les repas et au cours des $\mathrm{I} 2 \mathrm{~h}$ de repos alimentaire, donc en fonction de la réplétion relative du tube digestif, conduit à souligner chez nos Porcs l'allongement de la période de quiescence en un même site au fur et à mesure que se prolonge 1e jeûne nocturne. Dans les deux cas, cet allongement en valeur absolue de la phase silencieuse répond, au moins pour les 75 p. Ioo proximaux de 1'intestin grêle, à une réduction relative de la durée des phases d'activité irrégulière. Celle-ci est parallèle à la réduction quantitative de la masse de digesta au fil du déroulement du transit, ou de l'éloignement du dernier repas. Une telle relation directe entre la durée des activités de pointes irrégulières et l'importance de la masse de digesta présents dans la lumière intestinale a été mise en évidence chez le Mouton par RUCKEBUSCH et BUENo (I977). Ces observations concordent parfaitement avec ce que l'on sait du rôle particulier de l'activité dispersée à l'égard du déplacement des contenus digestifs, tant chez le Chien, le Mouton ou le Veau (BUENo, FioraMONTI et RUCKEBUSCH, I975; DARDILLAT et MARRERo, I977) que chez le Porc (RuCkebusch et BUENo, Y976; RERAT, CoRrisg et LAPLACE, I978). C'est en effet au cours de la phase II des complexes qu'est assuré l'essentiel de la propulsion des matériaux alimentaires.

\section{Effet de la digestibilité du régime}

Dans le cas particulier du quart distal de l'intestin grêle, l'allongement de la durée des cycles $(+40 \mathrm{mn})$ résulte, chez le Porc consommant un régime standard, à la fois de l'augmentation des durées de la quiescence $(+20 \mathrm{mn}$ ) et de 1'activité irrégulière $(+20 \mathrm{mn})$. Chez l'animal au régime amidon de maïs, l'allongement des séquences n'est que de $20 \mathrm{mn}$ sur l'iléon et est le seul fait de la plus grande durée de la phase de quiescence. L'ampleur de cette différence dans la durée (et 1'intensité) des activités irrégulières iléales est à relier au fait que l'iléon est, au moins chez diverses espèces de monogastriques, le siège d'une accumulation relative de digesta (L,APLACE, I975). C'est donc en ce site que les différences de digestibilité entre régimes pourront conduire aux plus grandes différences de la masse de matériaux alimentaires encore présents dans la lumière intestinale. Effectivement, chez le Porc équipé d'une fistule réentrante aux deux tiers de la longueur de l'intestin grêle, le régime standard conduit à collecter en $6 \mathrm{~h}$ après un repas d'épreuve un volume de digesta 2 à 3 fois plus important qu'après un repas d'épreuve quantitativement identique du régime amidon de mais hautement digestible (LAPLACF, I977; données non publiées).

Cette conséquence de la plus ou moins grande digestibilité du régime, à l'égard de la durée des activités irrégulières est en accord avec l'observation réalisée chez le Mouton par Ruckebusch et Bueno (I977) : la dérivation des digesta au niveau d'une fistule réentrante, sans restitution, entraîne une réduction de moitié de la durée de la phase d'activité irrégulière; celle-ci est à l'inverse considérablement accrue lors de perfusion additionnelle de digesta provenant d'un animal donneur. 


\section{b) Modifications liées à la composition du régime}

La vitesse de migration nettement plus faible enregistrée chez les Porcs consommant un régime amidon de maïs (ou S.F.), pour un nombre moyen analogue de complexes enregistrés en $24 \mathrm{~h}$ au niveau duodénal, constitue un fait nouveau dont le déterminisme reste à élucider. Une in fluence particulière des caractéristiques biochimiques du milieu intra intestinal dans son ensemble, ou de la quantité de tel ou tel nutriment libéré et absorbé dans un laps de temps relativement restreint doit être envisagée. La très grande rapidité de digestion enzymatique d'un amidon de maïs conduit à une hyperglycémie portale et même périphérique importante (RERAT, I975) et par conséquent à une très probable hyperinsulinémie. En effet, RERAT (I975) montre, pour un régime amidon de maïs rigoureusement identique à celui que nous utilisons ici, que l'absorption commence dès l'ingestion du repas; I50 $\mathrm{mn}$ après le début de celui-ci, la concentration des sucres réducteurs totaux (constitués pour $90 \mathrm{p}$. Ioo de glucose) est multipliée par 4 dans la veine porte, cependant que la concentration dans le sang artériel est portée du simple au triple.

Dans une telle situation qui appelle nécessairement une riposte insulinique, on fera le parallèle avec les effets décrits chez le Chien ou le Mouton. Chez ce dernier, l'insuline exerce une action excito-motrice à l'égard de l'électromyogramme intestinal, l'intervalle de récurrence des complexes atteignant 2 heures (au lieu de 60 à $90 \mathrm{mn}$ ) chez le sujet diabétique (BUENo et RUCKEBUSCH, 1975); la distribution en un seul repas à un mouton d'un aliment riche en nutriments directement assimilables induit une hyperinsulinémie et désorganise transitoirement le profil moteur intestinal (BUENO, WEEKES et RUCKEBUSCH, I977). Chez le Chien, 1'hyperinsulinémie post prandiale accompagne la disparition des complexes myoélectriques au profit d'une activité continue (RUCKEBUSCH, FIORAMONTI, I975), cet effet étant reproductible par injection d'insuline ou perfusion endoveineuse de $d$-glucose, $l$-leucine ou $l$-arginine (BUENo, Ruckebusch, I976).

Les éléments qui précèdent paraissent donc susceptibles de rendre compte également chez le Porc de la particulière activité post-prandiale consistant en une prédominance quasi-totale d'une intense activité de pointes irrégulière durant 2 heures. Ils pourraient également répondre de la relative désorganisation des activités de l'intestin grêle, traduite par l'augmentation très importante des migrations incomplètes ou des anomalies de migration. On voit mal par contre comment ces mêtnes phénomènes d'hyperglycémie/hyperinsulinémie post prandiales pourraient expliquer la lenteur de la propagation des activités de pointes régulières. Ce dernier fait ne peut qu'être enregistré, et sa permanence rapprochée de l'étalement, pour un niveau d'ingestion élevé, de l'absorption des sucres réducteurs résultant de la digestion sur environ $22 \mathrm{~h}$ (RERAT et al., I974; RERAT, I975). Il paraît ainsi probable que la quantité (et/ou la nature) des glucides simples libérés modifie certaines caractéristiques des complexes myoélectriques de façon indépendante des effets de la réplétion intestinale.

Accepté pour publication en mai 1978 .

\section{Remerciements}

Ce travail a bénéficié pour son achèvement de l'aide de la D.G.R.S.T. (Division du fond de la recherche) dans le cadre de l'action sur programme "Interrelations aliments - tube digestif " (Décision $\left.77 \begin{array}{lll} & 0463\end{array}\right)$. 


\section{Summary}

\section{Small boreel motility pattern and influence of feeding in pigs}

A systematic study of the characteristics of small bowel myoelectrical complexes according to feeding was made in Io Large White pigs. The intestinal electromyographic activities were recorded by means of supple chronical electrodes inserted under general anaesthesia. In all cases, the recordings were made after the animals had become accustomed to each of the 2 diets used in the experiment, i.e. a standard diet and a semi-synthetic diet including $82 \mathrm{p}$. Ioo purified maize starch. The feeds were offered in two meals, at $9.00 \mathrm{a} . \mathrm{m}$. and $4.30 \mathrm{p} . \mathrm{m}$.

Two to three myoelectrical complexes were permanently present along the pig small bowel. After administration of the standard diet, the average number of myoelectrical complexes per $24 \mathrm{~h}$ was 20.4 at the level of the duodenum, I6.6 in the jejunum and II.I in the ileum; $40 \mathrm{p}$. I00 of them did not migrate along the whole bowel. These values, steady for a given site in the same animal, varied much from one animal to another. The average recurrence interval ranged around $70 \mathrm{~min}$ in the duodenum, IIO-I20 $\mathrm{min}$ in the ileum. Although there were some irregularities in the migration of the complexes, the mean velocities recorded were $30 \mathrm{~cm} / \mathrm{min}$ in the duodenum, $10 \mathrm{~cm} / \mathrm{min}$ in the jejunum and $5 \mathrm{~cm} / \mathrm{min}$ in the ileum. The feed intake was responsible for the occurrence of a particular post-prandial organization of the motility for about 2 hours. During this period, the phase of quiescence was abolished, the irregular spiking activity was predominant without suppressing, however, the regular activities. The increase in the degree of intestinal repletion led to an intensification of the irregular activities; conversely, the length of the quiescence period increased during the nocturnal eating pause.

In comparison with the above data, the values recorded during $24 \mathrm{~h}$ after intake of the semi-synthetic diet were the following: 18.5 myoelectrical complexes in the duodenum, I 3.7 in the ileum. This resulted in an ileal recurrence at shorter intervals (90-I Io min). But, 50 p. Ioo of the regular activities in the ileum did not result from a continuous proximo-distal migration. The migration of the myoelectrical complexes seemed to be rather disorganized after intake of maize starch as compared with the recordings made after consumption of the standard diet. Parallel to that, substantially lower migration velocities were measured $(6.4 \mathrm{~cm} / \mathrm{min}$ at the proximal quarter of the small bowel). These particularities might be due to the composition of the diet, a hypothesis which is discussed in connection with the absorption of the digestion products of maize starch. The important lengthening of the quiescent phase between the duodenum and the ileum, to the detriment of the irregular spiking phase, may be related with a smaller digestive repletion (relative to the same intake level) depending on the high digestibility of the maize starch diet.

\section{Références bibliographiques}

BARONE R., 1976. Anatomie comparée des Mammifères domestiques. T. 3 : Splanchnologie, Fœtus et ses annexes. F. I Appareil digestif; appareil respiratoire. ${ }^{\text {re }}$ partie, chap. 6, p. $3^{85}$.

Borgida L. P., LAPLACE J. P., I977. Fvacuation gastrique comparée de régimes à base de fécule de pomme de terre crue ou extrudée chez le Porc en croissance. Ann. Zootech., 26, 585-593.

Bueno L., Froramontr J., Ruckebesch Y., 1975. Rate of flow of digesta and electrical activity of the small intestine in dogs and sheep. J. Physiol., Londres, 249, 69-85.

Bueno L., Ruckebusch Y., I975. Effets de l'insuline sur l'activité électrique du jéjunum chez. le Mouton. C. R. Soc. Biol., 169, 430-434.

BUENO L., RUCKEBUSCH M., I976. Insulin and jejunal electrical activity in dogs and sheep. An. J. Physiol., 230, $1538-1544$.

BUENo L., WeEkes T. E. C., RuCkebusch Y., 1977. Effects of diet on the motility of the small intestine and plasma insulin levels in sheep. Ann. Rech. Vet., 8, 95-104.

CARLSON G. M., BEDI B. S., CODF C. F., I972. Mechanism of propagation of intestinal interdigestive myoelectric complex. Am. J. Physiol., 222, I027-I030.

CODE C. F., MARLETT J. A., 1975. The interdigestive myoelectric complex of the stomach and small bowel of dogs. J. Physiol., Londres, 246, 289-309.

Dardillat C., I975. Gastro-intestinal motility in calf neonatal disease, in: RUTTER J. M., Perinatal ill health in calves, pp. II I-I 22, Simpsons Press, Newburg, Berkshire (U. K.). 
Dardillat C., Marrero E., 1977. Étude de l'électromyogramme global chronique de la paroi intestinale du veau préruminant : migration des phases d'activité régulière et relation avec le transit. Ann. Biol. anim. Bioch. Biophys., 17, 523-53o.

FAyart G., Daburon F., LAPlace J. P., I978. Étude d'un électromyographe numérique multivoies. Note technique C.E.A. (CEA-NT-55).

Grivel M. L., RUCKebUSCH Y., I 972. The propagation of segmental contractions along the small intestine. J. Physiol., Londres, 227, 61 I-625.

Hei, emans J., Vantrappein G., Janssens J., Peeters T., i977. Effect of feeding and of gastrin on the interdigestive myoelectric complex in man. 6th Int. Symp. Gastro int. Motility, Edinburgh, September 1977.

LAPLACE J. P., I968. Sur les phénomènes mécaniques et électriques du tractus digestif chez le Mouton. Th. Doct. Physiol. anim. Lyon, I vol, 200 p.

LAPLACE J. P., I972. Motricité gastro-intestinale chez le Porc. Étude descriptive par électromyographie et corrélations nutritionnelles. Rec. Med. I'et., 148, 37-6r.

LAPLACE J. P., I975. Le transit digestif chez les Monogastriques, revue : 2 /Phénomènes moteurs et mouvements des digesta. Ann. Zootech., 24, 489-55 I

LAPLACE J. P., GERMAIN C., I976. Endogenous and feeding induced periodicities of duodenojejunal fast activities in the Pig (Myoelectric migrating complex). I' $I$ in fth Congr. Int. Pig. Vet. Soc. June 22-24, Ames (Iowa).

LAPLACE J. P., TOMASSONE R., I970. Évacuation gastro-duodénale chez le Porc. Fistulation chronique par voie thoracique extra-pleurale. Recherche d'une technique d'analyse mathématique de l'évacuation. Ann. Zootech., 19, 303-332.

LATOUR A., I973. Un dispositif simple d'analyse quantitative de l'électronnyogramme intestinal chronique. Ann. Rech. Vet., 4, 347-353.

RERAT A., I975. Mesure quantitative in vivo de l'absorption chez le Porc. Application aux sucres et aux acides aminés, in: Réanimation entévale à faible débit continu. I.N.S.E.R.M. Ed., 53, $47^{-62}$

Rerat A., Aumaitre A., Vaissade P., Vauglíadf P., i974. Mesure de l'absorption de glucides au cours de la digestion de l'amidon de mais chez le Porc. C. R. Acad. Sci., 279 D, 83 I-834.

RERAT A., CoRring T., LAprace J. P., i978. Quelques aspects des recherches en physiologie digestive chez le Porc. Applications possibles. Journées Rech. porcine en France, I.N.R.A.I.T.P. Paris, 95-I 8 .

RUCKEBUSCH M., FERrF J. P., I973. Origine alimentaire des variations nycthémérales de l'activité électrique de 1'intestin grêle chez le Rat. C. R. Soc. Biol., 167, 2005-20og.

Ruckebusci M., Fioramonti J., 1975. Insulino-sécrétion et motricité intestinale. C. $R$. Soc. Biol., 169, 435-439.

Rcckebusch Y., I972. Origine de l'activité rapide de l'électromyogramme chronique de la paroi intestinale. C. R. Acad. Sci., Paris, 275 D, I 407-I 4 Io.

RUckebusch Y., I973. L'électromyographie globale des muscles lisses à partir d'électrodes chroniques intrapariétales souples. Rev. Med. Vet., 124, I 407-1 434.

RUckebusch Y., BUENo L., I976. The effect of feeding on the motility of the stomach and small intestine in the pig. Br. J. Nutr., 35, 397-405.

RUCKEBUSCH Y., BUENo L., I 977 . The migrating myoelectrical complex of the small intestine. An intrinsic activity mediated by the vagus. Gastroenterology, 73, I309-I3I4.

Ruckebusch Y., Dakdilita C., Hatey F., I972. La motricité intestinale chez le veau nouveau-

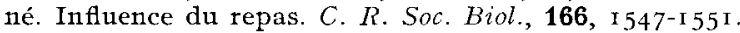

Ruckebusch Y., I.APLACE J. P., i 966 . Déclenchement expérimental de la rumination. $C . R$. Soc. Biol., 160, I I 2 -I I 7 .

RuckebUsch Y., LAPlace J. P., I967. La motricité intestinale chez le Mouton : phénomènes mécaniques et électriques. $C . R$. Soc. Biol., 161, 2517-2523.

Szurszewski J. H., rg69. A migrating electric complex of the canine snall intestine. Am. $J$. Physiol., 217, I 757 -1 763 . 